\title{
A new methodological approach for shoe sole design and validation
}

\author{
Miguel Davia-Aracil ${ }^{1,2}$ • Antonio Jimeno-Morenilla ${ }^{2 *}$ Faustino Salas ${ }^{1}$
}

\begin{abstract}
Shoe soles are extremely complex to design and manufacture due to their organically shaped but technically precise nature and their manufacturing constraints. Consequently, there is a need for the increased design process flexibility offered by the use of specific CAD methodologies and techniques, to facilitate the work of expert designers and permit effective construction of the three-dimensional elements comprising the complete structure. Recent advances in additive manufacturing systems have extended the possibilities of shoe sole design. These systems can be used to create the final mould and to incorporate dynamic elements that are of particular value in sports footwear. In this article, we present a new methodology for the design and validation of shoe soles. The methodology assists designers in the design concept process and in transfer of the design to manufacturing. The model incorporates both a structural and a functional approach. To this end, a set of specific tools have been developed that can be used to quantify design quality. For example, the model calculates the coefficient of friction, or slip resistance, necessary to comply with international standards concerning safety footwear.
\end{abstract}

Keywords: shoe sole design, additive manufacturing, rapid prototyping, parametric design, shoe slip resistance.

\section{Highlights}

- This paper presents a new methodological technique to design complex footwear soles.

- It shows how to specific CAD/CAE tools can improve footwear sole 2D/3D design shorting time and validating final model for manufacturing.

- It also shows how to additive manufacturing can be used in footwear industry for shoe sole mould production.

- A full practical case of shoe sole design is presented.

\section{Introduction}

\subsection{Shoe sole design}

Today, CAD/CAM systems face a number of new challenges and pressures created by market demand for low cost customised products as well as an increase in product quality [1]. Shoe soles are extremely complex to design and manufacture due to their organically shaped but technically precise nature and their manufacturing constraints. Hence, there is a need for modularity, scalability, reconfigurability, robustness and reliability that it is difficult for current CAD models, based on traditional legacy and rigid control structures, to achieve [2].

Three distinct parts must be defined in the design of a shoe sole. The first of these (see Figure 1a) corresponds to the upper surface in direct contact with the last; this part is critical for the correct fit of the model and is directly related to user comfort. The second part (see Figure 1b) forms the main body and defines the basic structure of the sole model as well as the initial aesthetic parameters that must be observed. The third part (see Figure 1c) is the outsole tread, which covers the entire lower surface in direct contact with the ground and is largely responsible for conferring the final product as a whole with its functional properties. The design of this latter part accounts for approximately $60 \%$ of the modelling carried out by a designer, not only due to aesthetic and functional reasons but also because it determines slip resistance and friction against the ground, as well as biomechanical behaviour.

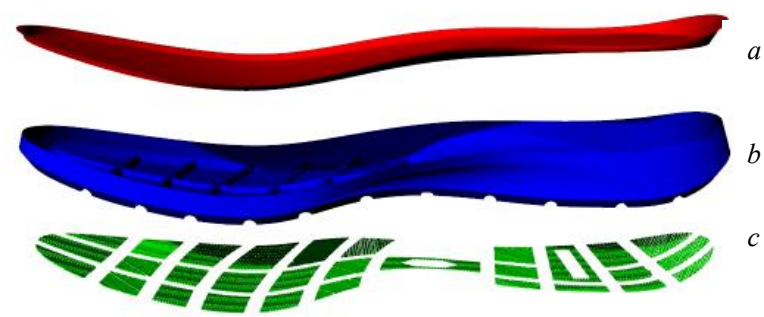

Fig. 1 - Parts of the sole: a) upper surface that fits to the last; b) main body; c) outsole tread

Within the current economic and industrial context, firms in the footwear sector that specialise in shoe sole manufacture are seeking to improve their product design and manufacture workflow and control in order to achieve cost, quality and time targets [3]. By incorporating 3D elements in CAD files, designers can include parameters and rules for adjusting model geometry using parametric and variational approaches (4D CAD) [4]. The goal is to reduce the time spent on repetitive design with no functional purpose, an activity estimated to account for $80 \%$ of the design process [5], to test different variations of the product, especially in the initial phase of the design process, and to improve product quality while reducing time and costs.

\footnotetext{
${ }^{1}$ Spanish Footwear Technology Institute (INESCOP), Polígono Campo Alto, 03600 Elda, Spain.

${ }^{2}$ Department of Computer Technology, University of Alicante Ctra. San Vicente del Raspeig S/N, 03690 Alicante, Spain.

*Corresponding author data:

Postal address: Ctra. San Vicente del Raspeig S/N, 03690, Spain Phone: +34 965903400 Ext. 2453

Fax: +34965909643

e-mail: jimeno@dtic.ua.es
}

The numerical simulations that were once used at the end of the design process to validate the resulting model 
are now being applied in the general design process, and especially in the initial stages, using and integrating parametric $\mathrm{CAD} / \mathrm{CAE}$ models. In the early stages of design, numerical simulation yields a better, faster design process $[3,6]$. This is particularly true in the case of shoe sole design, an area that is rapidly evolving towards the integration of design and simulation with increasingly complex models, and simulations in different areas of specialisation using diverse computational models that interact with each other. The vast amount of information involved in this process and the limited interconnection between modelling and simulation often lead to discrepancies between the data and model inconsistency [7]. Slip resistance simulation is a crucial task in the early stages of product development since it indicates to the designer whether to proceed with the model design or adopt a different approach in order to comply with international standards for slip resistance in safety or casual footwear.

\subsection{Common issues in shoe sole CAD software}

There are several generic 3D graphic design software systems on the market that are used in the footwear industry to design the $3 \mathrm{D}$ geometries that comprise the sole, including PowerShape ${ }^{\circledR}$, Rhinoceros ${ }^{\circledR}$ and SolidWorks ${ }^{\circledR}$. However, although these are suitable for designing the medium-high complexity free surfaces that comprise certain footwear components, they do not contain specific tools for performing the parametric design of the sole body and tread. Consequently, designing these complex structures with generic software is timeconsuming and requires qualified designers who have been trained in the use of specific tools at design schools of recognised standing in the footwear sector [26].

Outsole tread design in particular is highly complex because there are an unlimited number of possible designs. As can be seen in Figure 2, tread designs serve not only aesthetic but also highly functional purposes, especially as regards slip resistance.

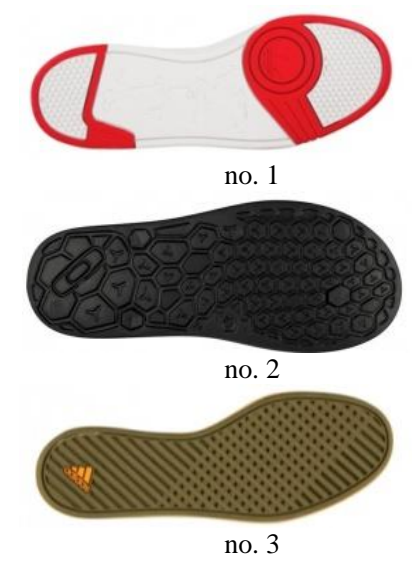

Fig. 2 - Sole treads

All of the above models require a multitude of $3 \mathrm{D}$ operations since, as can be seen, each of the treads incorporates a high number of geometries whose design entails a series of basic operations which must be repeated multiple times with exacting standards of precision. In addition, from a functional point of view, the design must comply with quality regulations and standards, especially in safety footwear. For example, model no. 3 might not meet current safety regulations since the outsole area is somewhat small; in this case, a calculation of the coefficient of friction would indicate whether or not the design is valid.

The usual procedure for designing a tread model begins with the creation of the basic $2 \mathrm{D}$ curves that define it, followed by their subsequent application to the body of the sole, which consists of a series of 3D surfaces. This procedure is performed in two stages: in the first, the $2 \mathrm{D}$ curves are projected onto the geometry, and in the second, the $2 \mathrm{D}$ curves are pasted onto the set of 3D surfaces. Generic systems cannot execute this second process, since it involves precise "unfolding" of a series of surfaces. An example of this process is depicted in Figure 3, where the model entails the design of a tread that extends upwards onto the lateral surfaces of the sole body. As can be seen in Figure 4 , the 2D curves necessary to create $3 \mathrm{D}$ tread should not be designed by means of projection but by "unfolding" a complex polysurface that transfers the set of $2 \mathrm{D}$ curves of the model to a $3 \mathrm{D}$ design space.

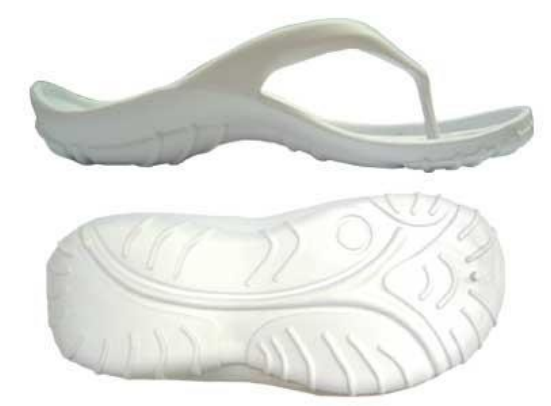

Fig. 3 - Tread design on a complex polysurface

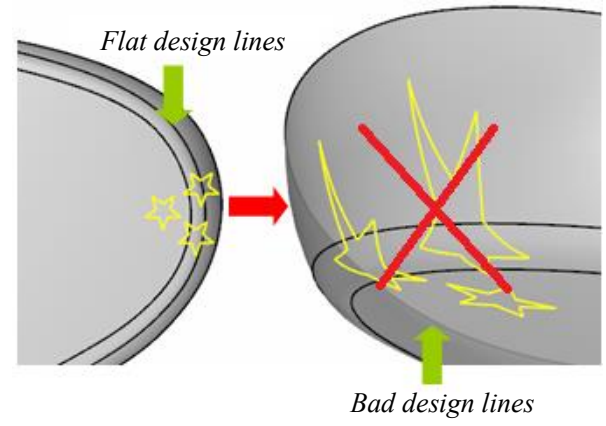

Fig. 4 - Error in the projection of curves on a polysurface

At present, shoe sole manufacturers employ basic design criteria to design non-slip models, often relying mostly on intuition and experience rather than quantitative measures of design quality due to the lack of specific tools capable of calculating and quantifying the friction generated by specific sole models.

\section{Research background}

In this section, we describe the main advances in $\mathrm{CAD} / \mathrm{CAM}$ that form the basis of the model proposed here. 


\subsection{Modelling for shoe sole design} modelling process.

\section{Parametric modelling}

\section{Surface unfolding techniques} mapping, or a combination of these.
The modelling techniques used in this research can be classified into three clearly defined groups: parametric modelling, used to speed up the design process; surface flattening techniques that define the shoe sole design; and lastly, polygonal mesh deformation techniques during the sole modelling process to model or adapt sole geometries to constraints selected by the user during the sole

The first parametric solid modelling systems arose as a specialisation of the constructive solid geometry (CSG) model [8, 9]. They incorporated the possibility of establishing reference parameters that defined the initial operations to apply to the objects to be modelled. For example, a user could define the radius and height of a cylinder before applying any subsequent editing operations to the model. In parametric CAD systems, shapes are initially generated on the basis of operations and constraints, and these latter also remain an integral part of model geometry while editing. Thus, the objects included in a given design present a dynamic behaviour. By contrast, the rules in non-parametric systems are imposed when the shapes are generated, but they do not apply once the shape has been added to the system: a line can be drawn perpendicular to another line, but the relationship between the two lines is not maintained by the system in the event that the orientation of either of the lines is changed.

Parametric modelling makes a significant contribution to design because it defines the semantic relationships between object representations [27]. These relationships define the necessary topological relationships between the objects that comprise a system, generating a graph of relationships that represent the interconnections between the different parts of a model. Thus, parametric modelling software applications specific to given design tasks (such as shoe soles) define relationships and constraints to express the logic of design conditions.

At present, surface flattening is based on prior discretisation of each surface. The result is a set of polygonal meshes that are associated with twodimensional parameterisation. In general, parameterisation will incur metric distortion, since only developable surfaces can be flattened on a plane without any deformation. Therefore, the objective of parameterisation is to find a bijective correspondence that preserves the original's geometric properties as much as possible. Examples include authalic (area-preserving), conformal (angle-preserving) and isometric (length-preserving)

Various recent studies on mesh deformation and modelling [19, 20] have formulated parameterisation as a problem of optimisation that encompasses both local and global elements. In essence, local transformations are sought that minimise the distortion of each triangle in the mesh, requiring that they are all linked by coherent 2D triangulation. The "as-rigid-as-possible" method of polygonal mesh deformation for editing purposes described in [20] is another of the techniques used for polygonal mesh parameterisation.

In shoe sole design, it is necessary to parameterise not only polygonal meshes but also the complex polysurfaces that define the shoe sole body, in order to apply different 3D textures or design lines to specific areas of the sole.

\section{Deformation techniques}

\section{a) Sketching}

Sketching-based geometric deformation of objects consists of editing curves and sketches of - or related tothe object to model or deform. Users can easily edit these curves, and the modifications are applied directly to the associated object, for example with the intuitive interface for mesh deformation presented in [10], multiple curves are sketched directly onto the object to be deformed; these reference curves are used to determine both the region of interest and the control of an individual deformation. Despite the generic nature of this method, it is mainly aimed at deformations that do not require much precision, such as those applied to large objects or elements. If a more detailed deformation is desired, such as eye shape or facial expression, the results are no longer adequate. To resolve this problem, reference and target curves were used in [11] to work with the specific area of the mesh necessary for facial articulation. A final approach worth mentioning in the field of sketch-based deformation is presented in [12]. In this case, the curves sketched onto the mesh to be modelled were used to create parametric curves which could be interactively manipulated with the consequent associated deformation of the surface. In [13], a variant of deformation known as curve network deformation is described, which uses the sketching techniques employed in the footwear industry to modify lasts. It is this type of solution which is used in the model presented here, since it yields good control of the object by the deformationcontrol curves, which take into account the geometry of the last onto which the shoe sole is fitted.

\section{b) Cage-based}

Cage-based deformation techniques were developed in response to the problems presented by free-form deformation (FFD) tools, while seeking to maintain the ease of use and speed of these methods. They consist of wrapping a low resolution polygonal mesh around the object to deform. This mesh has a similar shape to that of the object, whose vertices are represented as a combination of the cage vertices, multiplied by special weighting functions called coordinates. Manipulating the cage causes a smooth deformation in its interior that distorts the object.

This new approach was introduced in [14], based on the concept of mean value coordinates (MVCs), which define the functions of the weights governing the deformable space. MVCs for polygons were initially $-3-$ 
introduced in two dimensions as a means to achieve smooth information interpolations in generic polygons. Later, in [15], the method was extended by applying it to 3D triangular meshes. In [16], Laplace equations called harmonic coordinates (HC) were used to define the displacement of an object's vertices based on the deformation of the surrounding cage. Following on from the previous approaches, [17] introduced positive mean value coordinates (PMVC). Taking into account the advantages and disadvantages of each of the above methods, deformation is obtained without defects in concave areas (as with the $\mathrm{HC}$ ), but more rapidly and with less memory consumption than is the case with MVCs.

The main disadvantage of these types of approach is that the deformation does not necessarily preserve surface detail, since this problem is not addressed. To resolve this issue, the alternative presented in [18] obtains spatial deformation of an object while preserving its properties and detail. The method is based on green coordinates (GC, derived from Green's function theory), which are used to perform the deformation rotations necessary to ensure that the detail is preserved in the deformed object. The main advantages of cage-based deformation techniques are their simplicity, flexibility, and speed. Manipulating an object wrapped in a cage entails a small computational cost, since transforming a point requires a linear combination of the cage geometry using pre-calculated coordinates.

The model presented here is based on MVC deformation techniques because these make it possible to fit the sole, and they present better behaviour when the outsole tread area is modified, deforming the specific area in question smoothly and precisely.

\subsection{Additive manufacturing}

In the design for manufacturing (DFM) paradigm, designers must adapt their designs to the constraints of manufacture and/or to minimise the costs. However, recent improvements in rapid prototyping and additive manufacturing (AM) systems have provided the opportunity to rethink and redesign DFM methods in order to leverage the capabilities of these technologies. AM technologies offer new possibilities for customisation [21, 22], improving product performance, multi-functionality, and reducing overall manufacturing costs. More specifically, AM allows: a) shape complexity: it is possible to construct virtually any shape, assemble a range of elements in a single piece and customise geometries; b) material complexity: pieces can be manufactured with a combination of various materials and properties; c) hierarchical complexity: multi-scale structures can be designed and manufactured from microstructure through geometric mesostructure (sizes in the $\mathrm{mm}$ range) to macrostructure; and d) functional complexity: when parts are constructed using AM, the interior of a piece is always accessible. This makes it possible to integrate multiple functional aspects in the design. For example, functional mechanisms and integrated components can be manufactured directly to achieve multi-functional parts.

However, there is a lack of AM-related CAD tools in the footwear sector. The development of CAD methods and tools for AM in accordance with the DFM paradigm would yield a significant performance improvement in the design of pieces, allowing designers to fully leverage the possibilities offered by the materials and manufacturing processes. In terms of pieces, materials and hierarchical complexity capabilities, DFM could shift from an emphasis on minimising costs to a focus on achieving capabilities not previously feasible.

In [23], DFM additive manufacturing (DFAM) has been defined as the synthesis of shapes, sizes, geometric mesostructures, material compositions and microstructures to fully exploit manufacturing process capabilities and thus achieve the desired performance and other objectives related to product life cycle.

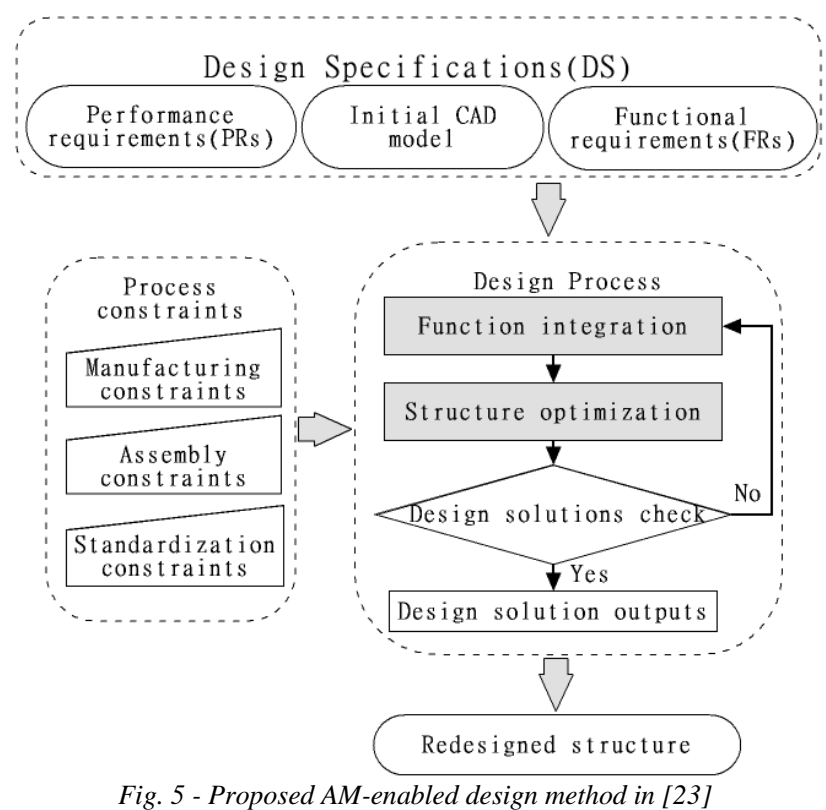

Here, we present a methodology for shoe sole design that is compatible with the design objectives and workflow of DFAM (see Figure 5). Additive manufacturing offers footwear manufacturers new possibilities for improving their market competitiveness. One of these is customisation, through the additive manufacture of aesthetic elements for subsequent insertion in the shoes. Another is the possibility of endowing the end product with functional properties that render it attractive to customers. From this perspective, concepts such as reusability, adaptability and efficiency are interconnected in the shoe sole design process, allowing designers to adopt a new approach to product design.

\section{Proposal for shoe sole design and validation}

Below, we present a new methodology for shoe sole design and validation. The process involves new $\mathrm{CAD} / \mathrm{CAE}$ tools that are primarily aimed at systemising each of the proposed steps and reducing the time required for design. This novel approach has been implemented and validated using commercial sole design software developed by the Spanish Footwear Technology Institute (Spanish initials: INESCOP), Icad3DP®. 


\subsection{Sole body design}

The first step in shoe sole design is to define the main body and the fit of the surfaces that will come into direct contact with the last used to manufacture the footwear model. Figure 6 depicts the complete process of creating the base surfaces of the sole body. The sole body (see Figure 7) is defined by a set of master lines which are interconnected by linking curves that define the surfaces comprising the body. These master lines and linking curves directly specify the dimensions of the sole to manufacture. The model constraints applied in the design include aesthetics and fit between sole and last. Sole design is based on the definition of master lines (see Figure 7a-b-c) and the subsequent creation of parametric linking curves (see Figure 7d), which define the sole body.
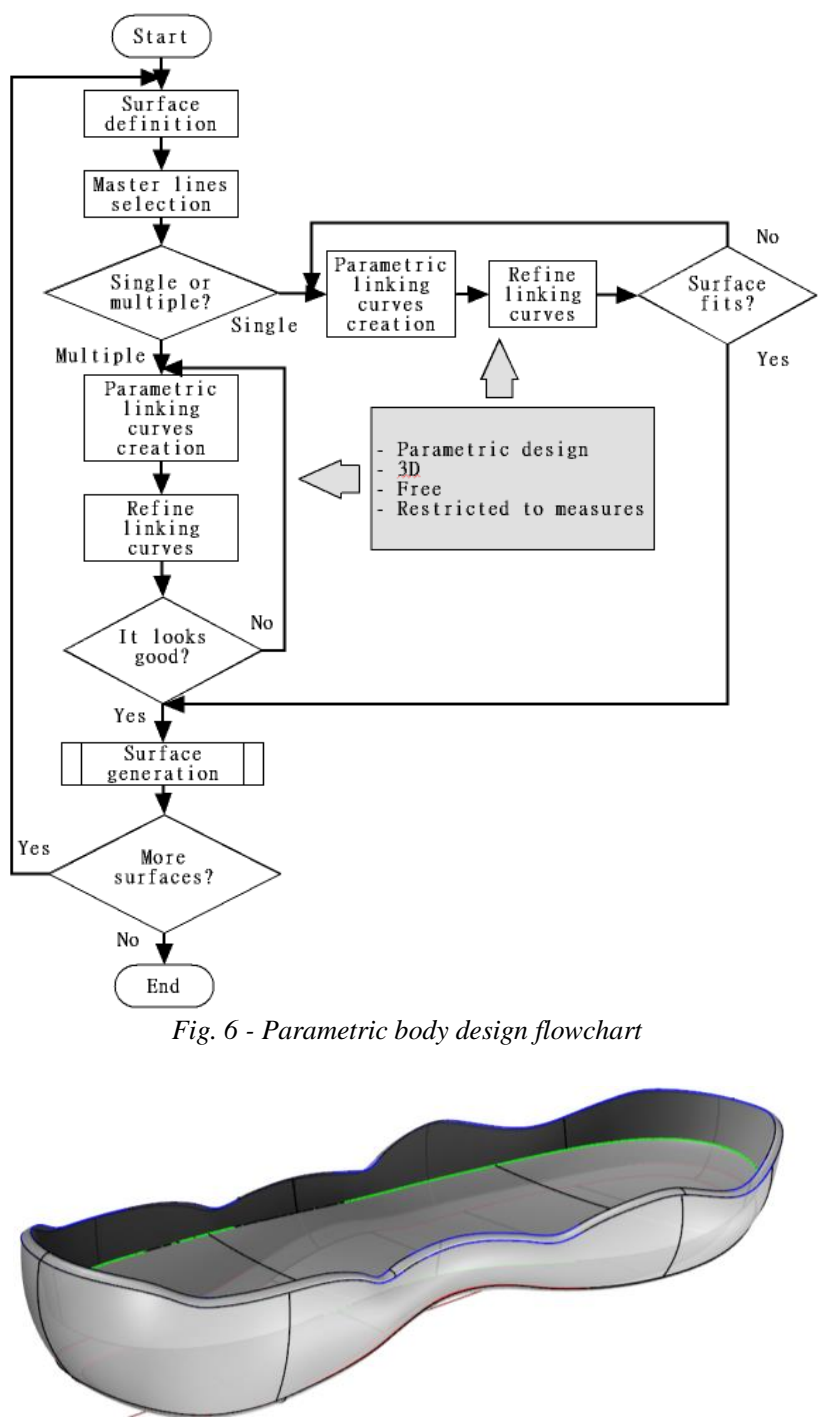

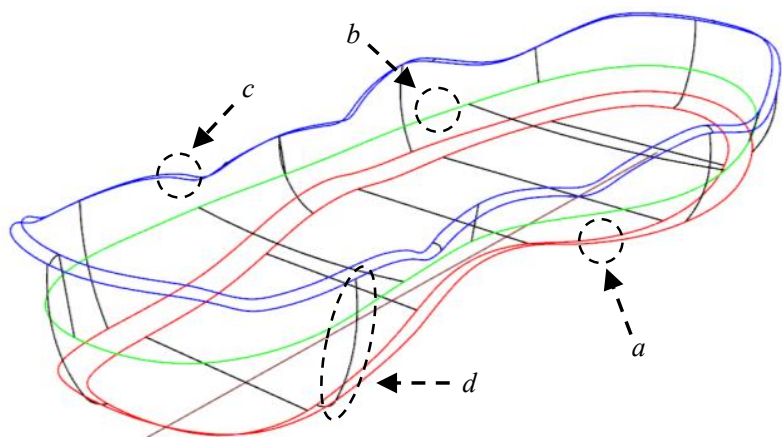

Fig. 7 - Master lines $a-b$-c, and linking curves, $d$

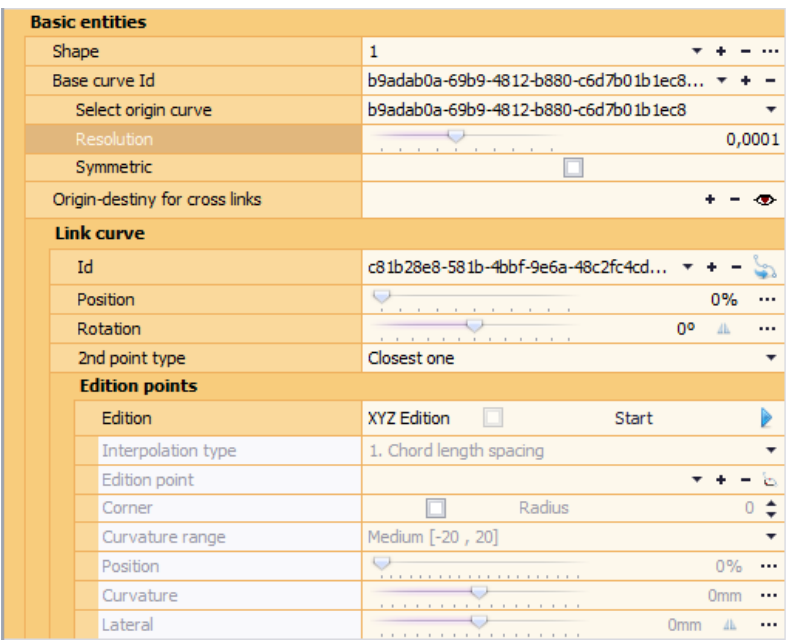

Fig. 8 - CAD tool for parametric links

Figure 8 depicts the CAD tool used to create parametric links from the master lines in order to precisely define the surfaces that comprise the model. This allows the user to modify links, adjusting their values according to the established specifications. The parametric link curve editing features allow the user to rapidly perform a series of operations such as: a) parametrically position, rotate and connect the link; b) create parametric editing points; c) precisely adjust editing points, curvature, position, and lateral displacement; and d) dynamically define editing point values.

Figure 9 provides an example of parametric link curve editing. Specific parameters used in the footwear sector for sole design can be defined for each editing point, such as curvature with respect to the straight line that joins the anchor points. As shown in Figures 10 and 11, it is also possible to specifically adjust editing points by entering Cartesian coordinates.

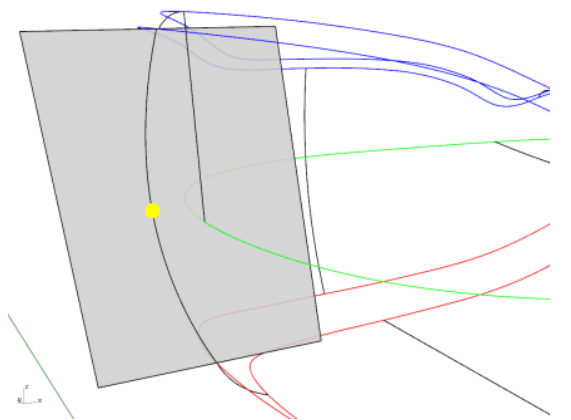

Fig. 9 - Parametric editing of links 


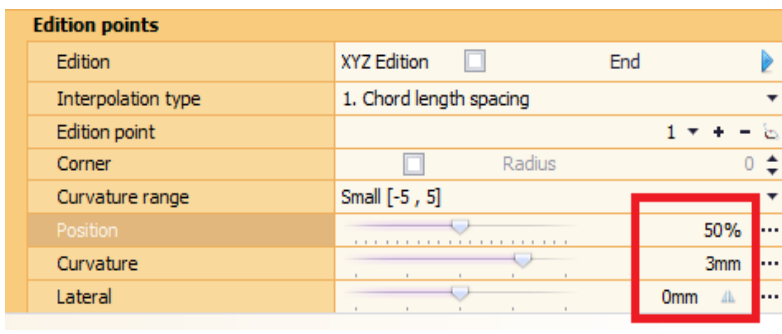

Fig. 10 - Editing point, design parameters

\begin{tabular}{|c|c|c|}
\hline \multicolumn{3}{|l|}{ Edition points } \\
\hline Edition & XYZ Edition & End \\
\hline Interpolation type & 1. Chord length spacing & - \\
\hline Edition point & & $1+-0$ \\
\hline Corner & Radius & $0 \leqslant$ \\
\hline Precision range & & $0,1 \div$ \\
\hline Working plane & Construction plane & 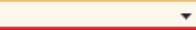 \\
\hline X Position & & $0,93 \div \nabla$ \\
\hline Y Position & & $12,97 \div \nabla$ \\
\hline Z Position & & $0,00 \div \nabla$ \\
\hline
\end{tabular}

Fig. 11 - Editing point, coordinates

Figure 12 shows how it is possible to define more complex operations with editing points, for example to create a corner point and control measures dynamically to verify that the position of the point is located correctly with respect to neighbouring points. In Figure 13, various parameters are defined which enable the user to position an editing point accurately in relation to surrounding points.

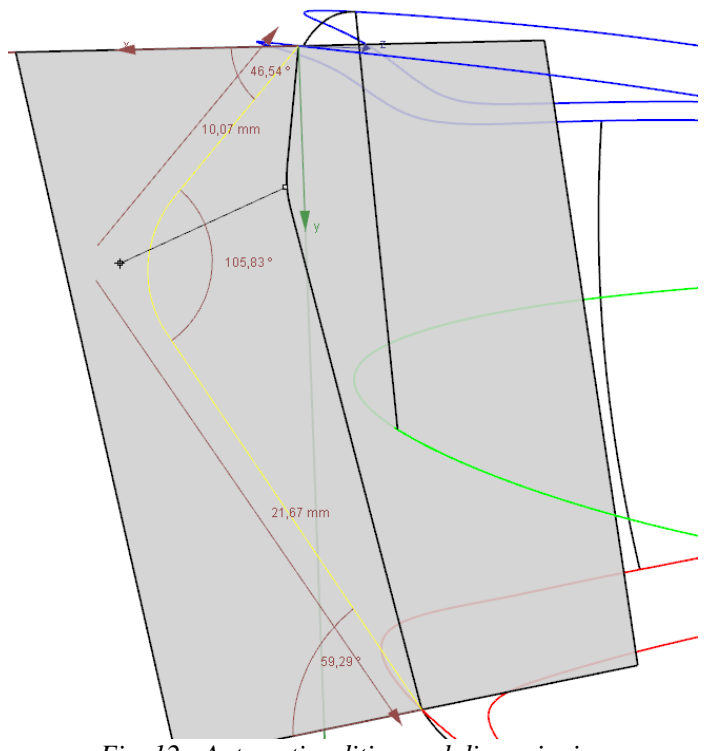

Fig. 12 - Automatic editing and dimensioning

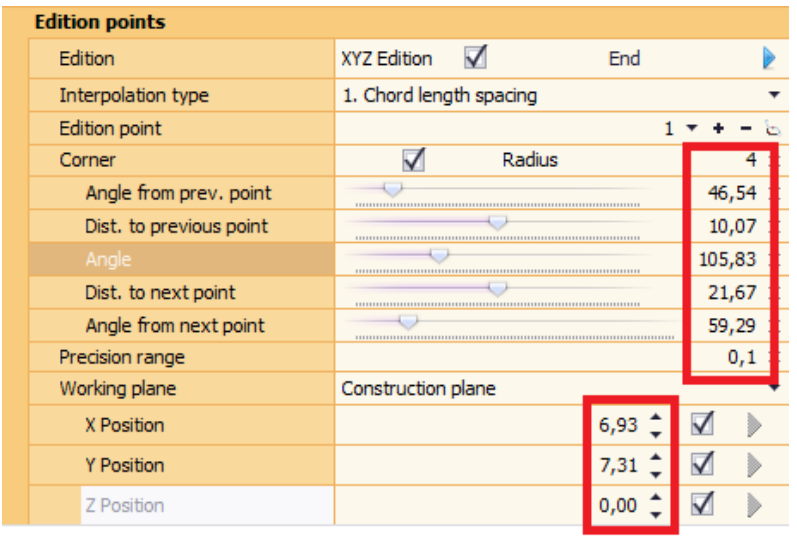

Fig. 13 - Editing point, extended parameters

To define the upper surface in direct contact with the last, the curvature of each control point is precisely defined and this curve is used to simulate the base and curvature of the last. The design of this area must be very precise, since it is necessary to ensure the fit with respect to the last. Figure 14 depicts the linking curve in the metatarsal area, and its position and curvature parameters are given in Figure 15.

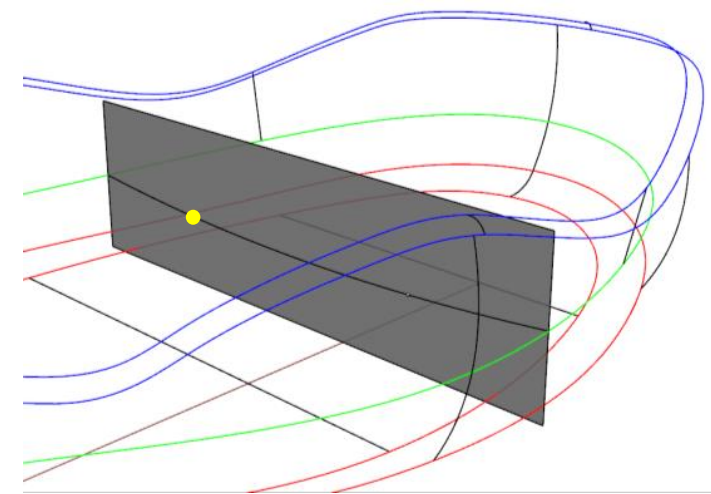

Fig. 14 - Link in the metatarsal area

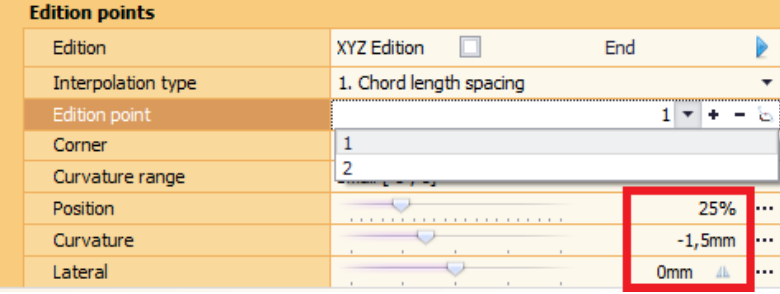

Fig. 15 - Editing point, position, curvature and lateral parameters

To facilitate the work of the designer, links can be generated in a range of ways, including: a) creation of nlinks; b) copying links; c) symmetrical copies of links; d) acquisition of links from the reference curve (reverse engineering). Linking curves can be defined by specifying different types of join between the first and second anchor points. As shown in Figures 16 and 17, there are various possibilities for obtaining the second point according to design needs.

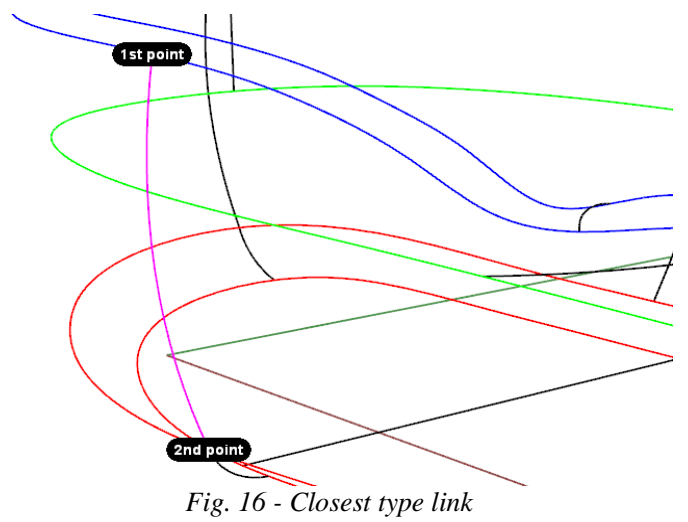

Fig. 16 - Closest type link 


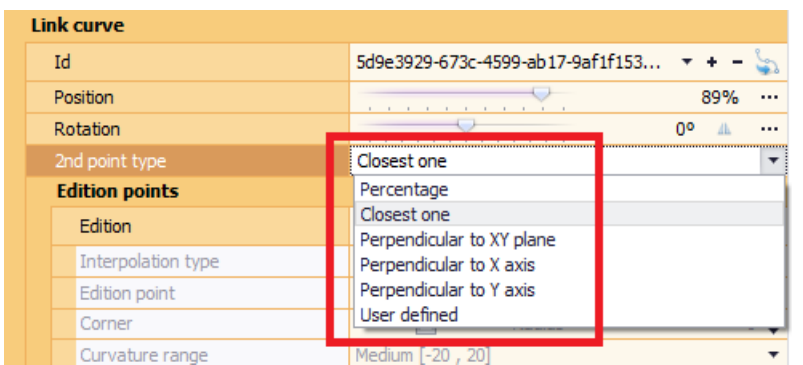

Fig. 17 - 2nd point type link

In addition, Figures 18 and 19 show how a parametric link can be configured, indicating its position and rotation. In this case, the linking curve is located in $89 \%$ of the perimeter of the base curve with a rotation of $6^{\circ}$.

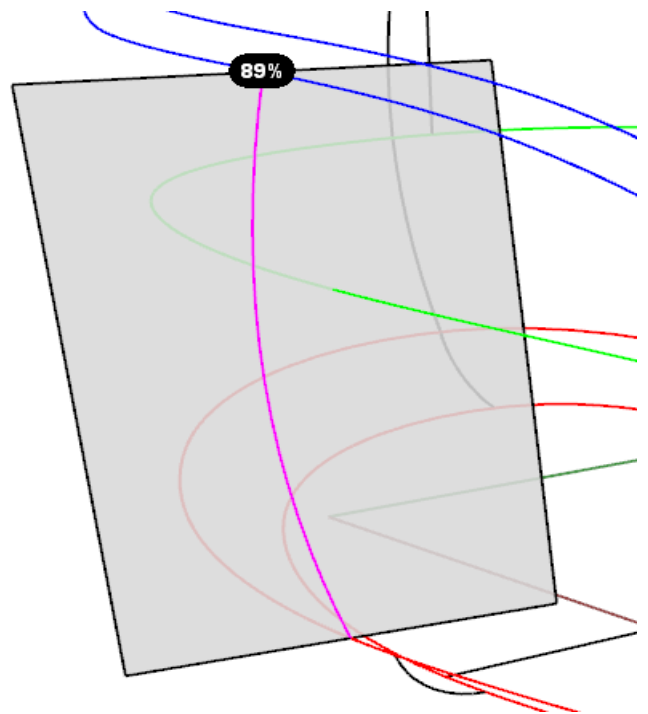

Fig. 18 - Parametric position of the link

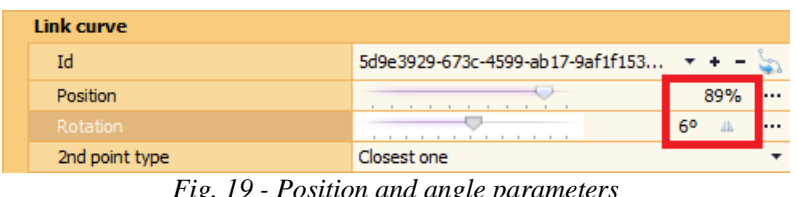

The possibility of creating a shape from different parametric surfaces is defined at a higher level. Thus, a sole body design is defined by a set of parametric surfaces. Each of these parametric surfaces is defined as shown in Figure 7 , and the number of initial surfaces that comprise the sole body design is variable.

\subsection{Sole tread design}

Sole tread design involves parametrically creating 2D designs that must adapt to the boundary curves defined by the designer. Repeated patterns are automatically created in specific areas, taking into account that they can parameterised by defining: a) type of distribution: linear, circular, or guide curve; b) mismatch with respect to the boundary curves; c) rotation and scaling; and d) elimination of small curves. Figure 20 shows the design methodology employed for this process. All of these functionalities are aimed at allowing designers to create various $2 \mathrm{D}$ tread design alternatives in the minimum time possible. Performing this task manually involves relatively intense post-processing of the resulting geometries and thus entails high costs, which are reduced by the use of this specific tool. Figure 21a shows the boundary curves to be filled with the patterns depicted in Figure 21b. Figure 22 defines the CAD tool that parameterises the design and allows the designer to switch easily between the different distribution possibilities. This tool yields a significant reduction in the time taken to produce a $2 \mathrm{D}$ tread design, allowing the designer to rapidly create multiple variations and accurately validate the designs generated.

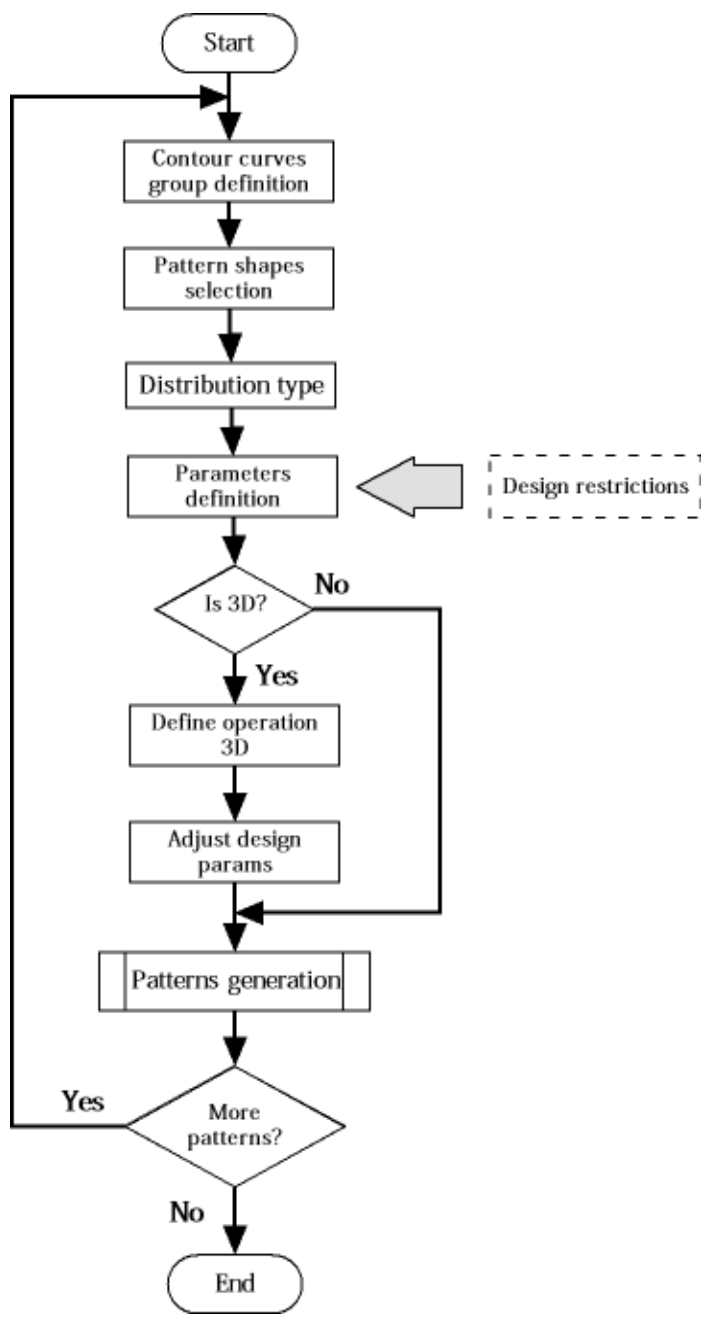

Fig. 20 - Parametric $2 D$ tread design flowchart

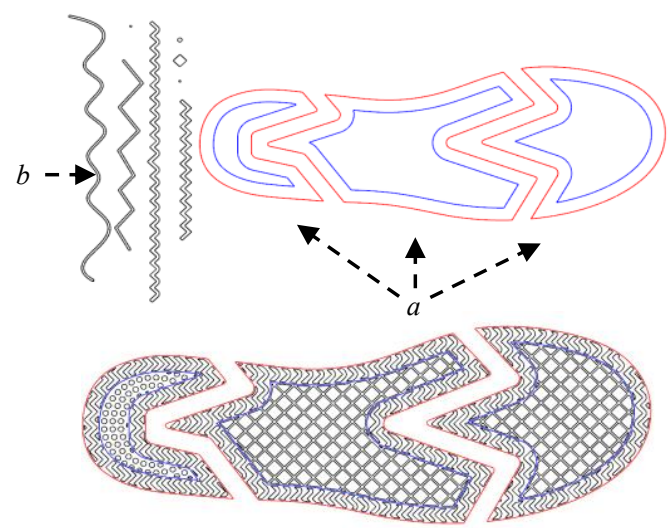

Fig. 21 - Curves: a) boundary; b) pattern 


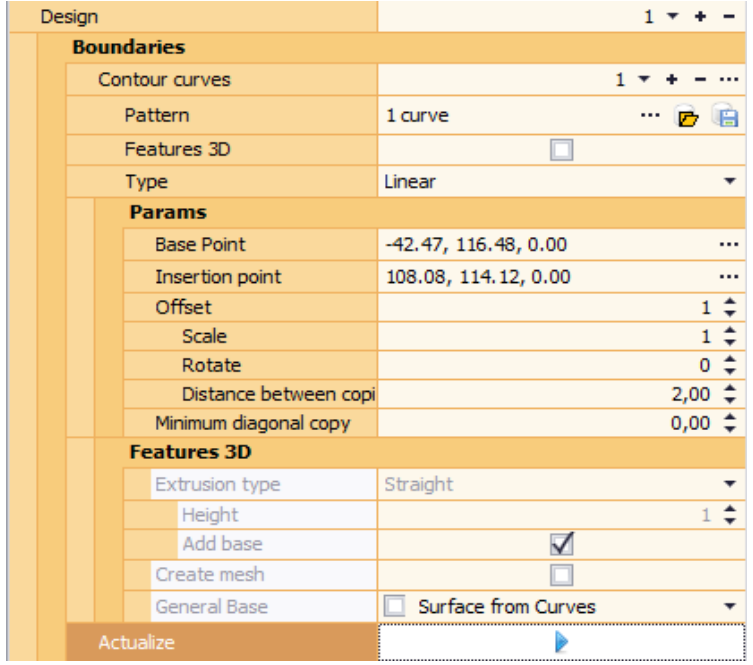

Fig. 22 - Tool parameterisation

3D tread designs can be created rapidly from a multilevel hierarchical system of 2D curves, facilitating the work of the designer. To do this, a series of levels are established that indicate the $3 \mathrm{D}$ operation to perform with the curves at each level, defining a series of parameters such as: a) positive and negative height, indented or raised relief; b) mismatch of the curves.

The design methodology employed is shown in Figure 23. Figure 24 depicts two hierarchical levels, the first formed by the curves in Figure 24a, with $2 \mathrm{~mm}$ of indented relief and an offset of $1 \mathrm{~mm}$, and the second formed by the curves in Figure 24b, which are dependent on the first level and have a relief height of $2 \mathrm{~mm}$. Figure 25 shows the two hierarchical levels created, together with the parameters offered by the tool. The hierarchical definition of these $3 \mathrm{D}$ design levels allows designers to change given parameters dynamically in order to control features such as different heights. Similarly, it is possible to rapidly change the $2 \mathrm{D}$ design curves used and automatically redesign the final tread in 3D for subsequent validation of the design. This yields a considerable reduction in design time, especially for tread model designs using repeated patterns, which can account for between 30 $40 \%$ of the total models a company may generate in one season. In addition, as will be seen in section 3.4 , this is directly linked to slip resistance validation.

The aim of polysurface parameterisation is to create complex 2D designs on these surfaces and then transfer them easily and accurately from $2 \mathrm{D}$ to $3 \mathrm{D}$, allowing the designer to perform the following operations: a) design, transferring 3D curve and geometry details; and b) texturing, transferring 3D geometric texture details. The methodology employed for this process is shown in Figure 26. Figure 27a shows the polysurface for parameterisation, yielding two polygonal meshes, the 3D mesh (Figure 27b) and the corresponding flattened 2D mesh (Figure 27c). Figure 28 shows the transfer of 2D tread design curves (Figure 27d) to 3D geometry. The next step is to perform the $3 \mathrm{D}$ curve operations necessary to conclude definition of the $3 \mathrm{D}$ sole tread design.

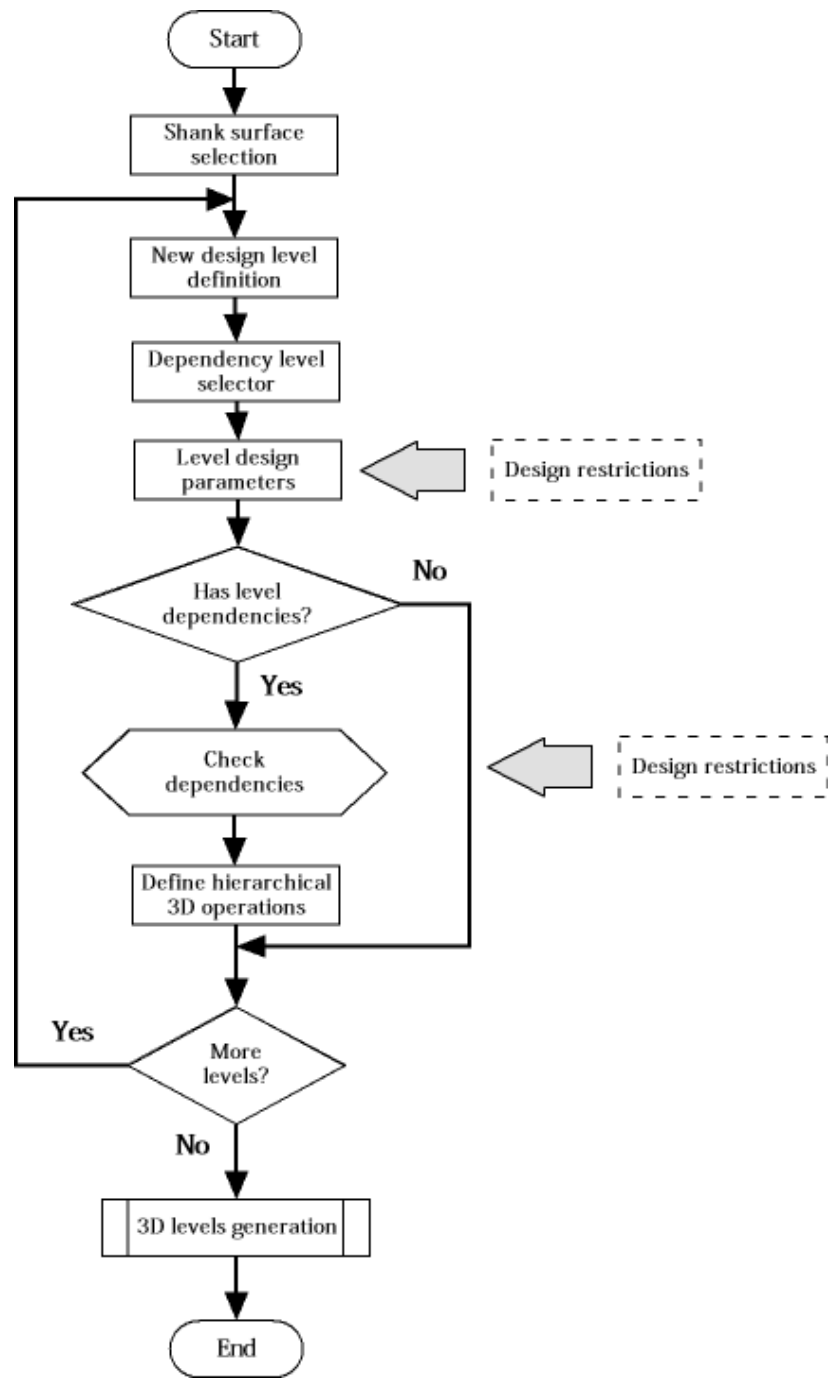

Fig. 23 - Parametric 3D tread design flowchart

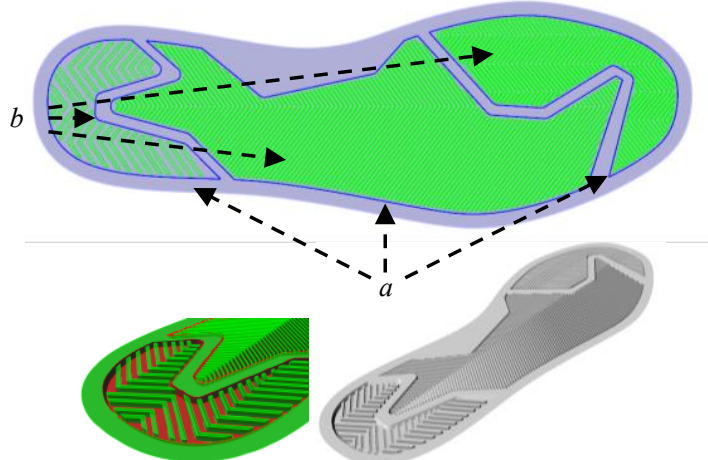

Fig. 24 - Hierarchical 3D tread design: a) level 1; b) level 2 


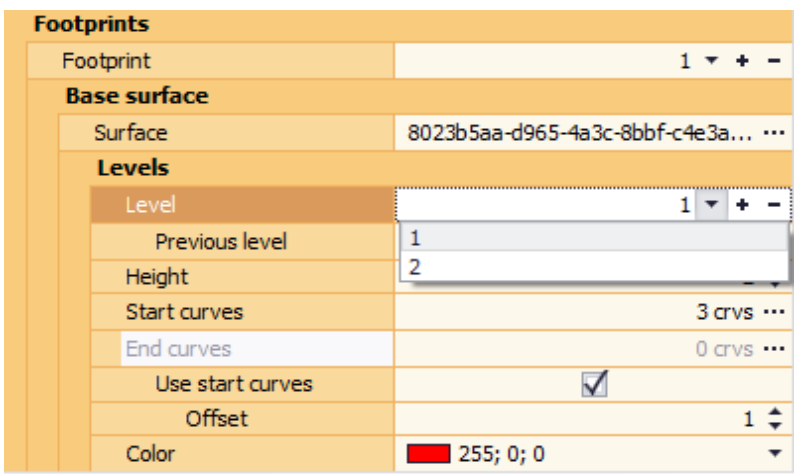

Fig. 25 - Tool parameterisation

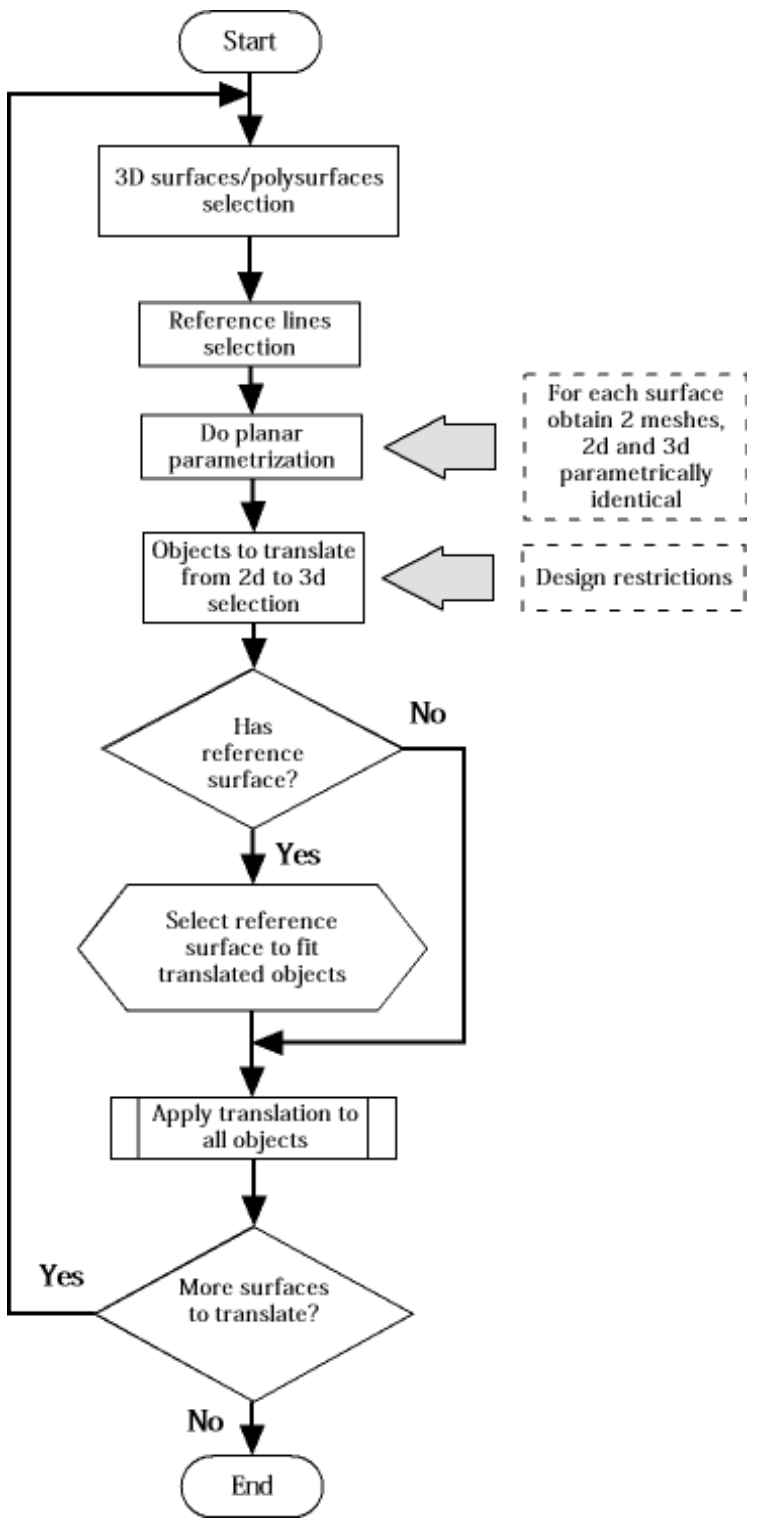

Fig. 26 - Parametric flattening and 2D/3D transfer flowchart

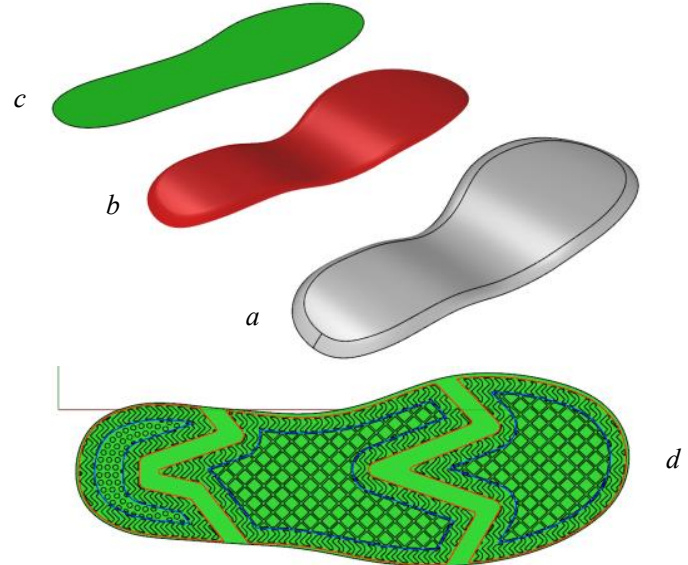

Fig. 27 - Parameterisation of polysurfaces: a) $3 D$ polysurface; $b$ ) $3 D$ mesh; c) 2D mesh unfolding; d) $2 D$ design curves on flattened $2 D$ mesh

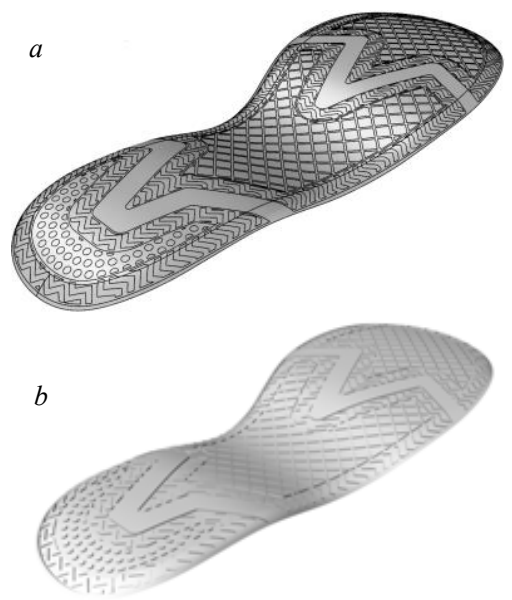

Fig. $28-2 D / 3 D$ tread design: a) curves pasted onto polysurface; $b$ ) final $3 D$ design

\subsection{Sole fitting}

One of the main reasons for changing a sole model once its design is complete is when a change has been made to the last on which it was designed. When this happens, the designer needs to perform the necessary modifications to ensure that the model will fit. This normally entails starting afresh and redefining the design according to the new master lines in direct contact with the last. With this in mind, the present proposal incorporates deformation techniques that make it possible to modify an existing 3D sole design rather than having to repeat earlier work.

The tools developed for this purpose deform the model using two different techniques: a) curve network [23]; b) cages. Both incorporate specific options of great use to shoe sole designers, enabling them to create a correct fit. The process employed is depicted in Figure 29. Figure 30 illustrates an example where the sole must be modified because the curve that defines the sole of the last has been changed. Using a pair of curves, the original curve (Figure 30a) and the target curve (Figure 30b), the tool is configured to deform the area indicated by different shading (see Figure 31a-b), depending on the needs of the design. Figure $32 \mathrm{a}-\mathrm{b}$ shows the deformed sole, now slightly longer and wider than the original, and Figure 33 gives the CAD tool parameters. 

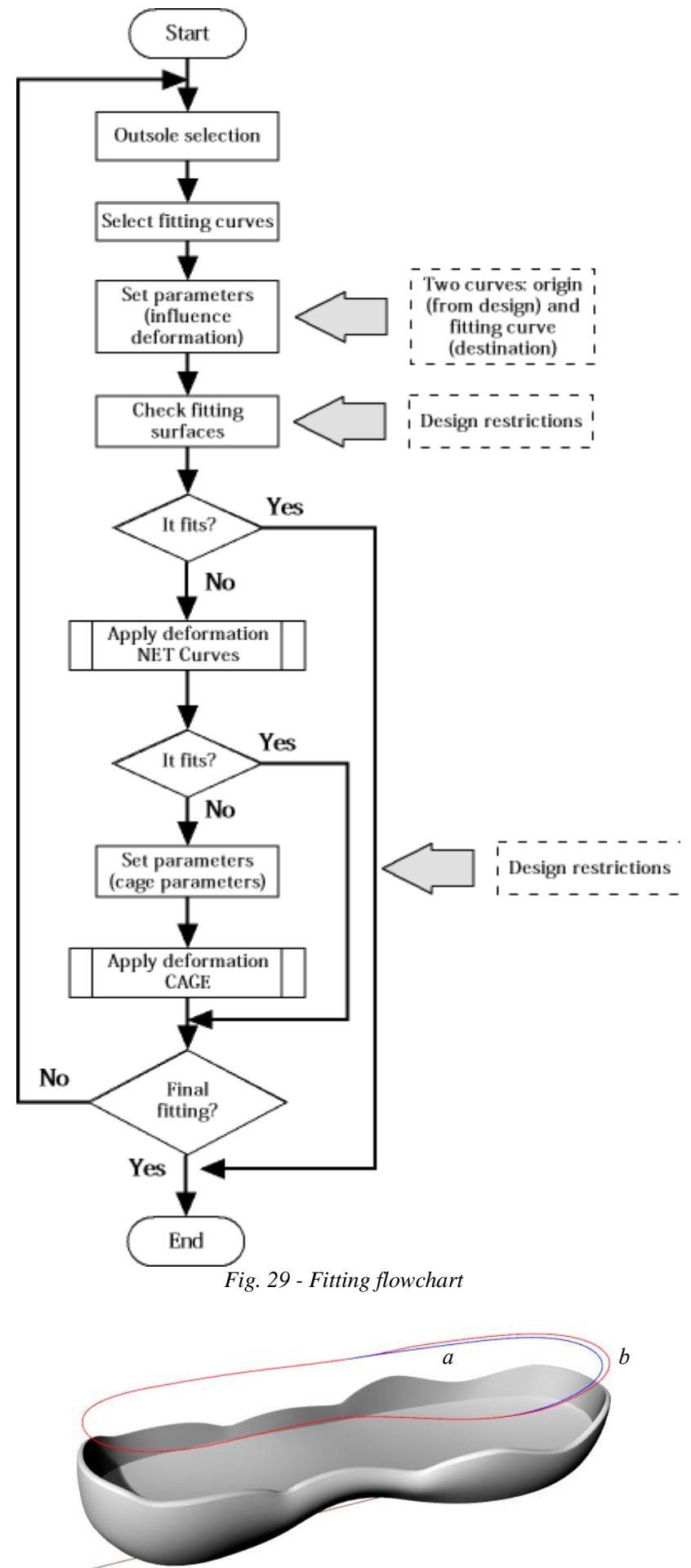

Fig. 30 - Deformation curves: a) original curve; b) target curve

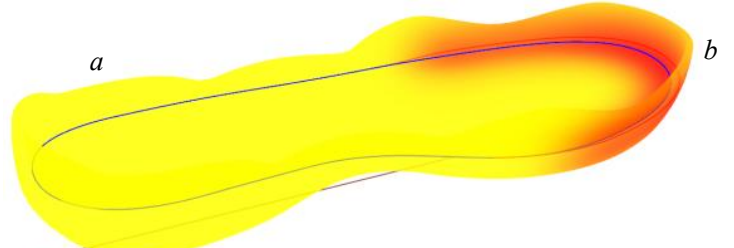

Fig. 31 - Deformation effect: a) non-deformable area; $b$ ) deformable area

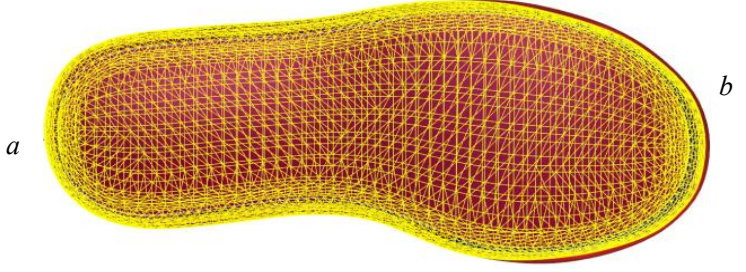

Fig. 32 - Deformed sole: a) before deformation; $b$ ) after deformation

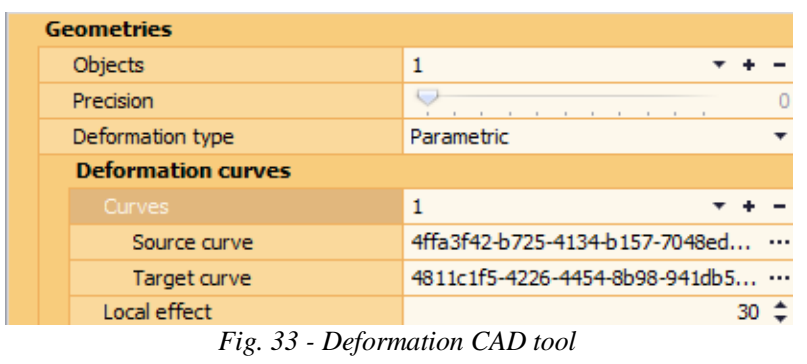

Figure 34 illustrates an example of cage-based deformation. Two types of cage are defined, a polycube cage and a close-fitting parametric cage, with greater or lesser resolution depending on the type of deformation to perform. Figure 35 shows the CAD tool and parameters required to configure the polycube cage. It is possible to create different types of cage which, depending on the parameters applied, can be configured to allow better deformation of the sole.

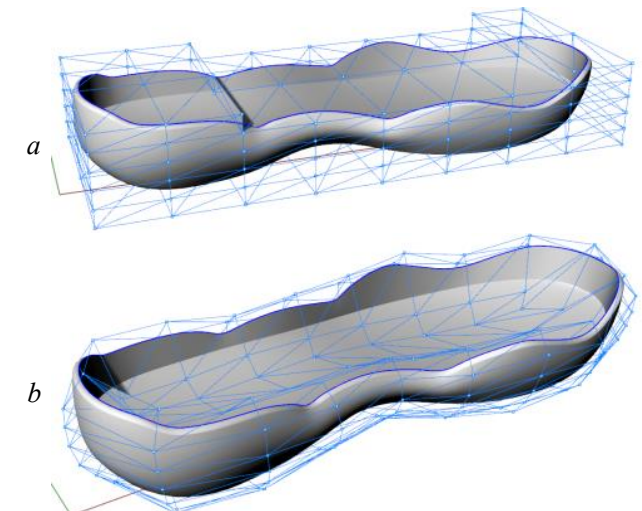

Fig. 34 - Different types of cage: a) polycube cage; b) close-fitting parametric cage

\begin{tabular}{|c|c|}
\hline \multicolumn{2}{|l|}{ Geometries } \\
\hline Objects & +-5 \\
\hline Precision & $15 \square$ \\
\hline Cage type & Polycube \\
\hline \multicolumn{2}{|l|}{ Parameters } \\
\hline Cage & c1a6d35f-ffe4-4597-84ae-96ec82... \\
\hline No. $X$ cubes & $7 \div$ \\
\hline No. $Y$ cubes & $2 \div$ \\
\hline No. $Z$ cubes & $2 \div$ \\
\hline
\end{tabular}

As can be seen in Figure 36, the cage vertices are manipulated to deform the geometry, obtaining the result shown in Figure 37. Using the method described in [15], a smooth deformation is produced in the area affected by the selected vertices, creating a modified version of the sole that complies with the new constraints imposed by manufacturing needs. 


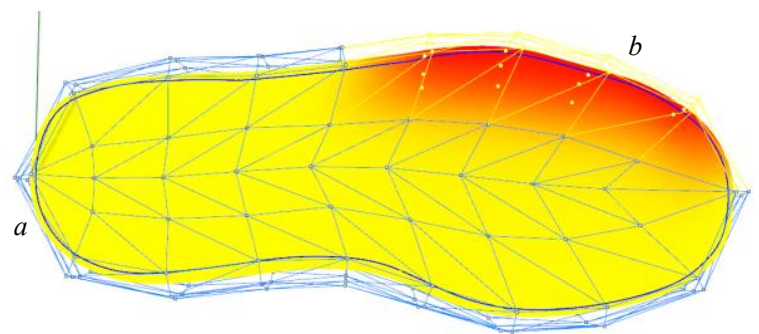

Fig. 36 - Area of influence: a) non-deformable area; b) deformable area

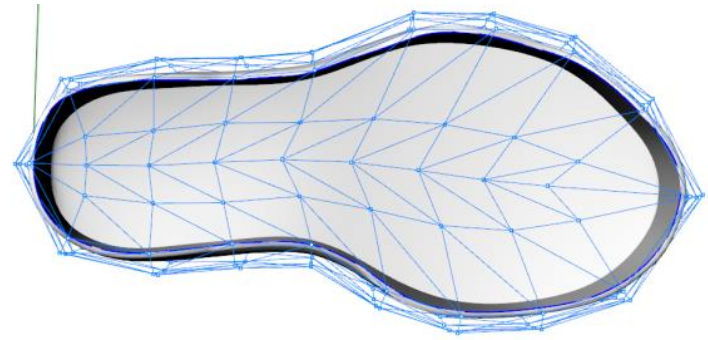

Fig. 37 - Result of deformation

\subsection{Sole design validation}

Currently, shoe sole manufacturers use basic design criteria to design non-slip models, and often rely on intuition and experience. The lack of CAD tools that can be used in footwear design is the main problem that designers face when designing a non-slip model. The CAD tools employed in previous stages of sole design can be used to calculate the coefficient of friction $(\mathrm{CoF})$, rendering the creation of prototypes cheaper, faster and more effective, and obtaining parameters for shoe friction on a given floor surface, for both professional and daily use. In the ULTRAGRIP project [24], working guidelines and specific mathematical models have been developed to calculate slip resistance without conducting an actual physical test (which would entail real manufacturing and the associated costs). The main results of this project have been to simulate slip behaviour using predictive mathematical models of the coefficient of friction, and to formulate guidelines for improving product slip resistance. Meanwhile, in the DEMOULTRAGRIP project [25], a set of CAD tools have been developed that incorporate predictive mathematical models that are fully integrated with the usual shoe sole design workflow.

The proposed model (see process in Figure 38) uses predictive mathematical models of the $\mathrm{CoF}$ based on parameters that characterise the design of the created sole: type of shoe (professional or street), type of material, hardness of material, heel height and area of contact with the ground. This latter parameter, contact surface $\left(\mathrm{cm}^{2}\right)$, determines the $\mathrm{CoF}$ prediction; simulations have shown that the larger the contact surface, the greater the slip resistance of the design.

By way of example, Figure 39 depicts a real sole model while Figure 40 shows the CAD model generated in the initial stages of design; at this stage, the design is created rapidly, since only the outsole tread is considered in the simulation.
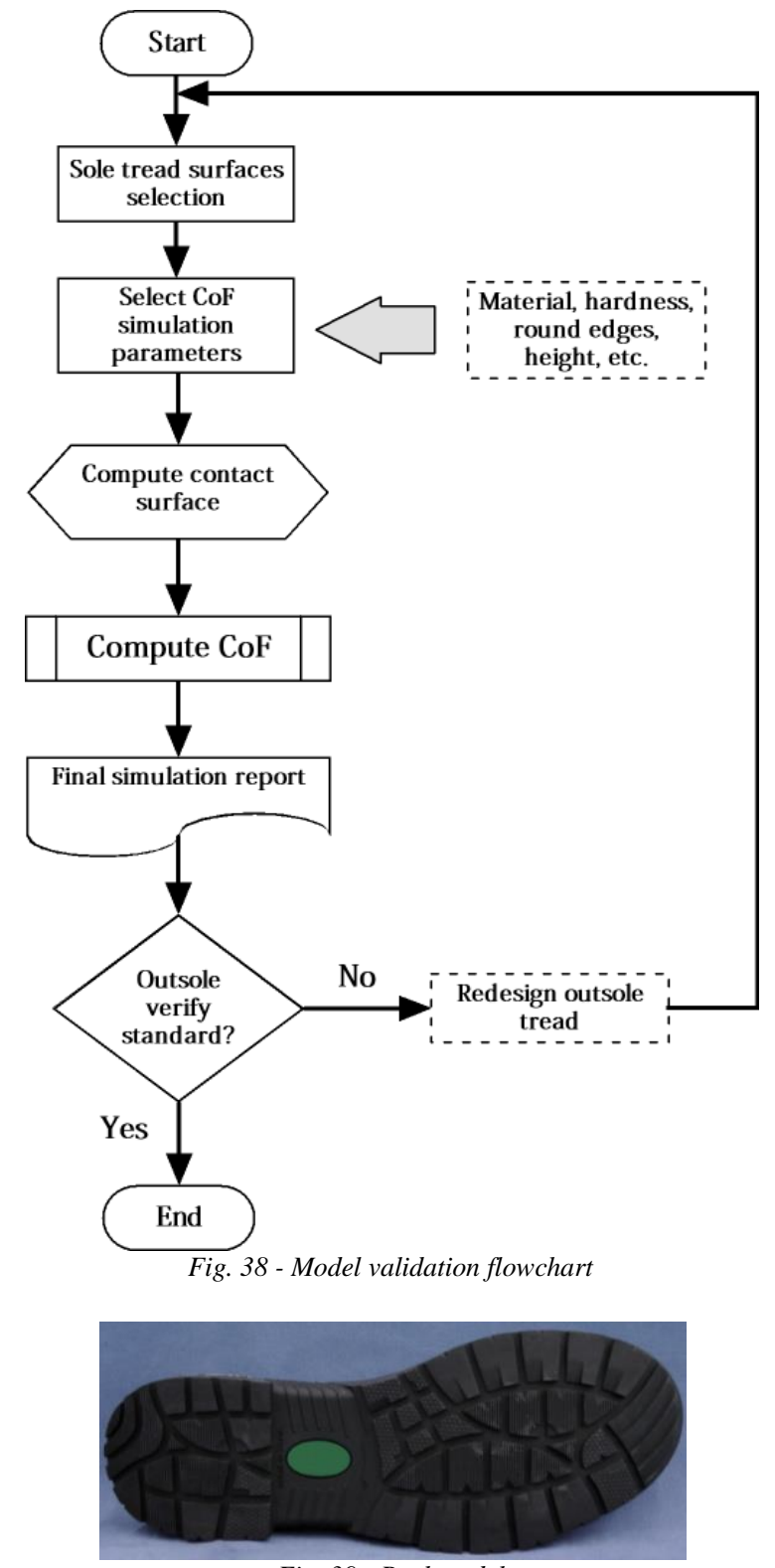

Fig. 39 - Real model

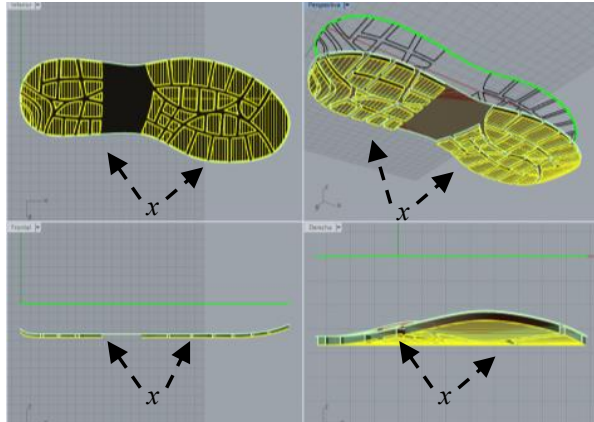

Fig. 40 - CAD model: areas marked with an " $x$ " come into direct contact with the floor surface

As shown in Figures 41 and 42, the footprints indicate the contact surface area in different cases: flat, heel or ball footfall. The CAD tools shown below estimate and determine the area of contact in each of these situations to be tested, in accordance with current legislation and with minimal user intervention, using the 3D CAD model. 
The contact surface is calculated on the basis of the outsole surfaces selected by the designer which come into direct contact with the ground. To simulate the pressure exerted by the sole on the ground (in accordance with European regulations for the real test EN-ISO 13287), $500 \mathrm{~N}\left(\mathrm{~N}=\mathrm{kg} \mathrm{m} / \mathrm{s}^{2}\right)$ of pressure is applied perpendicular to the ground. This value is simulated after several experiments to generate a contact plane that "cuts" the surfaces and defines the area of contact with the ground. Figure 43 shows the sole surfaces that are in contact with the ground (Figure 43a); this plane intersects and cuts the surfaces (Figure 43b), yielding the simulated contact area. Figure 44 shows the result of the simulation, with the parameters of this particular model and the final validation report.

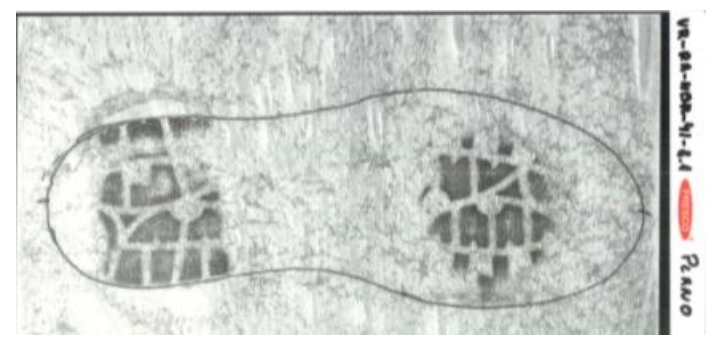

Fig. 41 - Footprint of the real model: flat

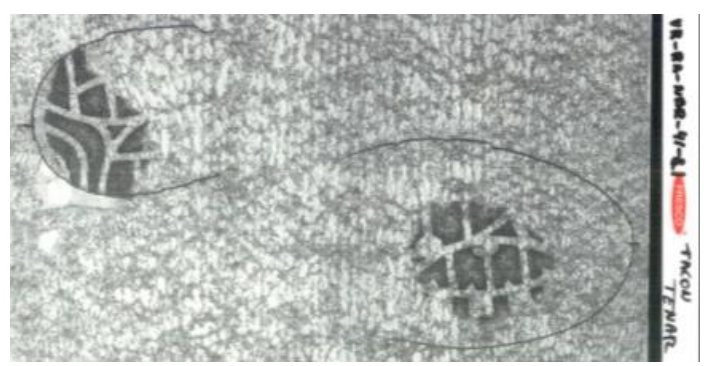

Fig. 42 - Footprint of the real model: heel, ball

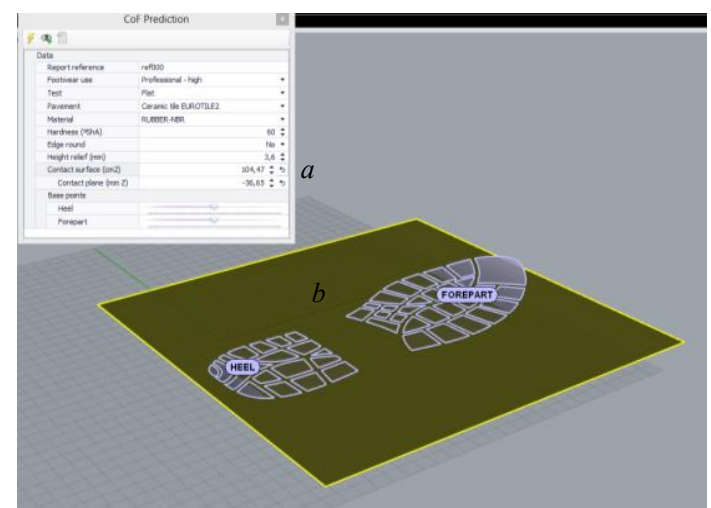

Fig. 43 - CAD parameters: a) contact surface; b) sole surfaces in contact with the ground

\subsection{Additive manufacturing}

Additive manufacturing techniques and 3D printers are used in the shoe sole industry for several purposes; to manufacture the mould into which the final sole is injected, and more recently, for product customisation [28] and to add new functional properties. Thus, there are several additive manufacturing applications in the shoe sole industry (Figure 45).

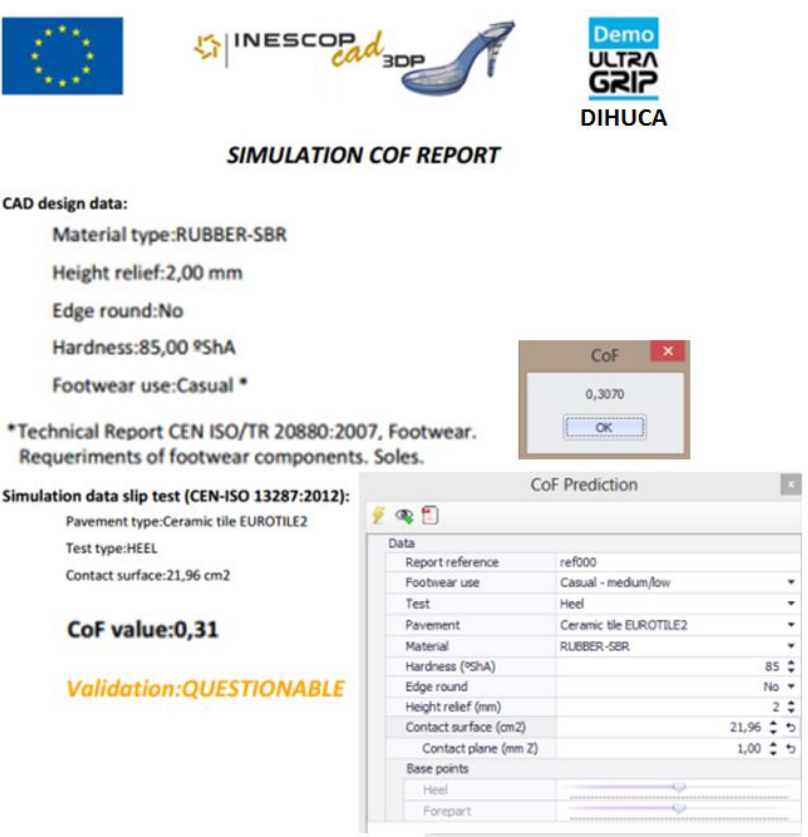

Fig. 44 - Simulation results and $\mathrm{CoF}$

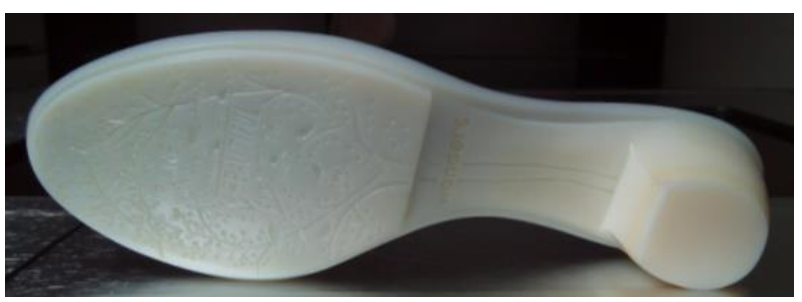

Fig. 45 - 3D print of a sole

\subsubsection{Mould manufacturing}

Additive manufacturing techniques and 3D printers have been used to manufacture the moulds into which the final soles are injected. Once a CAD prototype has been created, it is printed in 3D for use as the basis of an aluminium mould, which is manufactured employing traditional foundry techniques because CAM machining using 3-axis CNC machine tools is not viable. This basic aluminium mould represents the main body of the sole and must be completed with the addition of an upper cover.

Additive manufacturing is particularly useful when the sole model is highly textured, especially on the lateral surfaces of the body or the outsole tread. In these cases, the use of additive manufacturing and the process described above is essential. As can be seen in Figures 46 and 47, the sole model illustrated presents textures that cannot be machined in the mould using 3-axis CNC. Therefore, it is necessary to manufacture a physical prototype by means of $3 \mathrm{D}$ printing in order to then manufacture the mould using traditional techniques (see Figure 48).

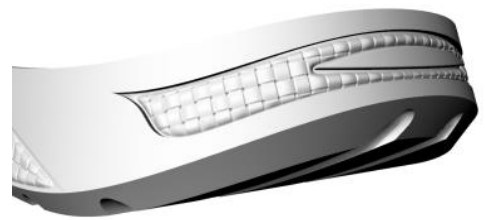




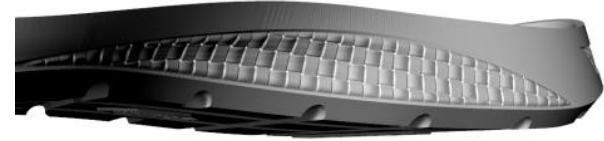

Fig. 46 - 3D textures: rear and lateral
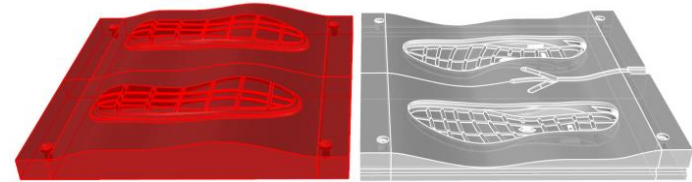

Fig. 47 - On the left, upper cover. On the right, lower cover

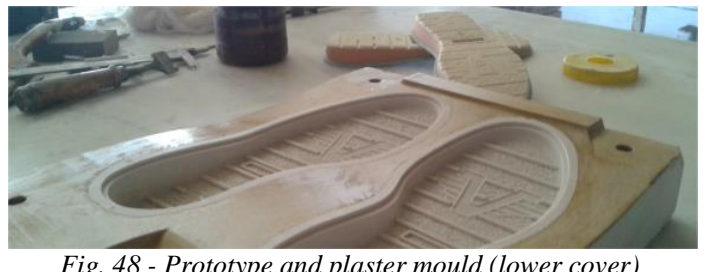

\subsubsection{Adding functional elements}

A level of structural design and the capacity to dynamically insert given elements is important with some sole components, particularly in sports footwear. Various footwear brands are incorporating 3D parts in the soles, either for aesthetic or specific functional purposes. Figures 49 and 50 depict examples of dynamic reconfigurable elements that can be incorporated into soles. A parametrically reconfigurable element is shown, which has been used in this model to increase the contact surface in a given area of the sole when the wearer is walking on different ground. The element can be added and removed, and since it is printed by additive manufacturing, it is easily replaceable in the case of wear or breakage.

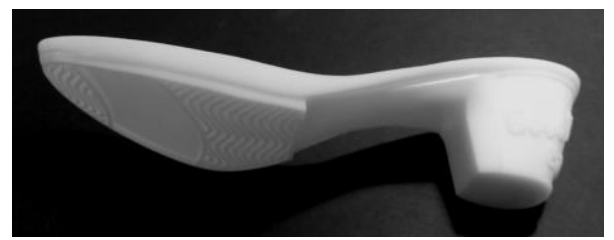

Fig. 49 - Main sole body

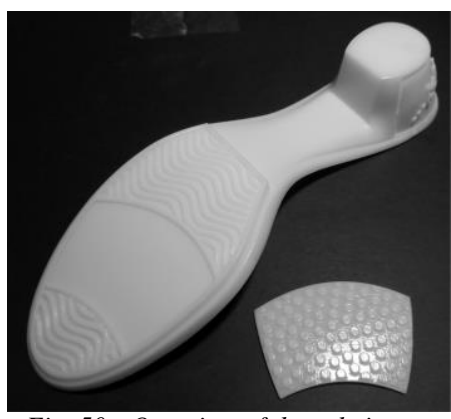

Fig. 50 - Overview of the sole insert process begins with the initial lines created by the designer, which are considered master lines for the sole body design, using the parametric design CAD tool (see Figures 51 and 52).
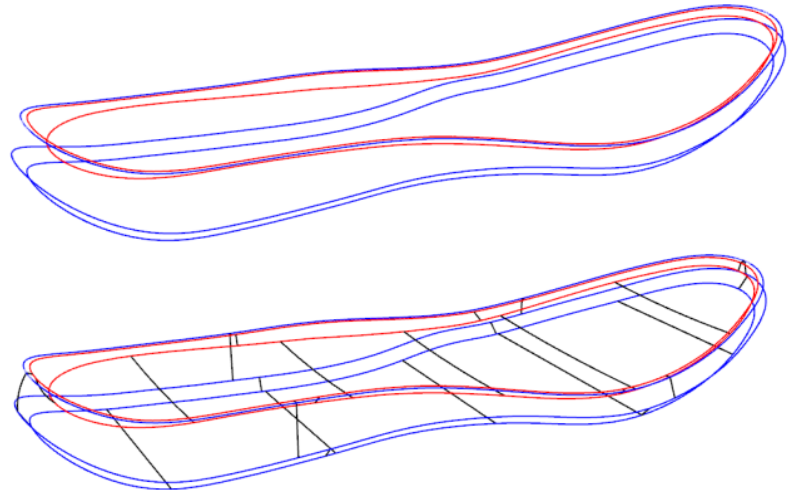

Fig. 51 - Master lines and parametric links

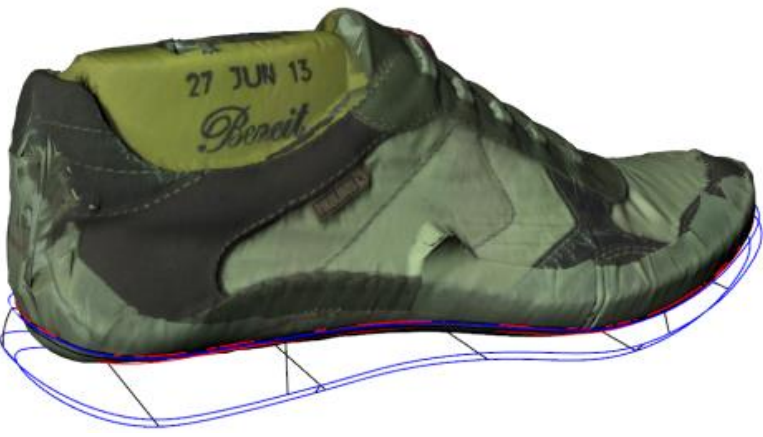

Fig. 52 - Last fitting

Once the sole body has been designed, it is necessary to design the outsole tread. In this case, we will show the tread design on a 3D polysurface; consequently, prior to designing the $2 \mathrm{D}$ tread, the polysurfaces must be flattened where the tread design lines will be applied. Once the corresponding polysurfaces have been flattened and the 2D tread lines designed, the 2D information will be transferred onto the 3D polysurfaces to be used for modelling (see Figures 53 and 54).

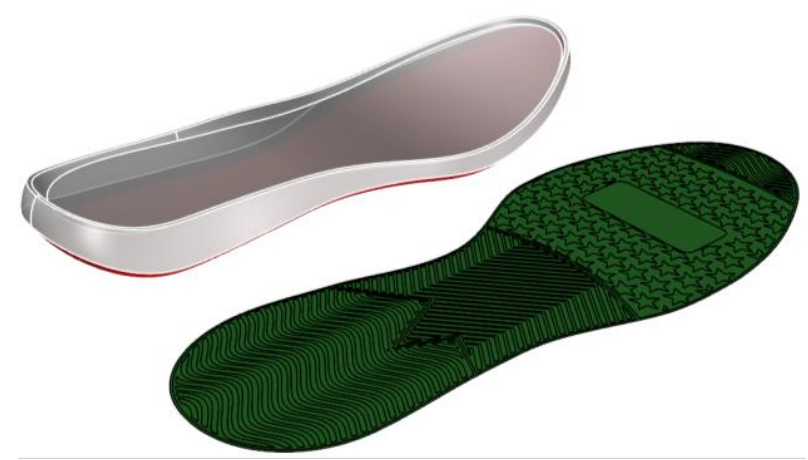

Fig. 53 - Flattened polysurface with design curves

\section{Case study}

Below, we will describe the complete design of a shoe sole, using the methodology presented in this paper. The estimated times for each phase are shown in Table 1. The 


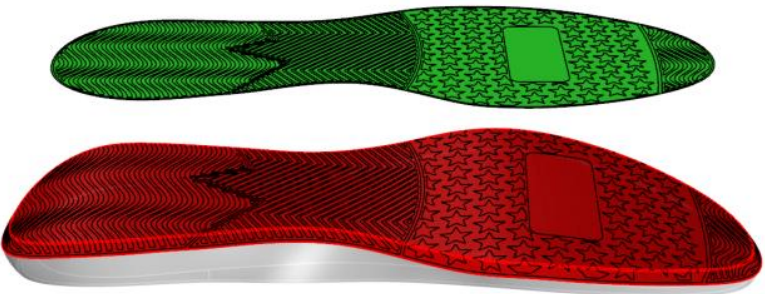

Fig. 54 - Curves transferred onto the polysurface

The next step is to create the corresponding 3D shapes by means of extrusion and surface trimming (see Figures 55 and 56).
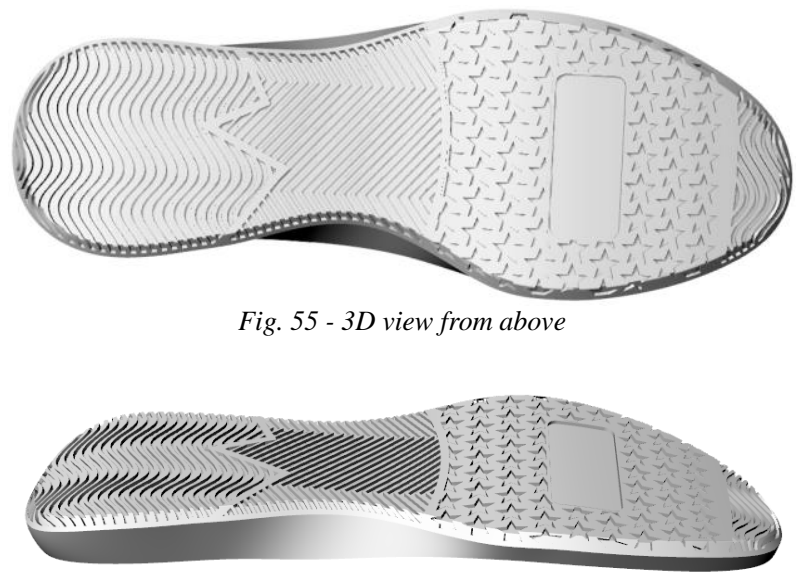

Fig. 56 - 3D perspective view

This 3D tread design can be created using the multilevel hierarchical design tool. For the present example, this tool has not been used since the characteristics of the model render it unnecessary.

At this point, it is possible to simulate the model's coefficient of friction in order to analyse whether or not the tread design is valid. This is performed using the CAE simulation tool, which indicates whether the model is in compliance or not with current legislation (see Figures 57 and 58).

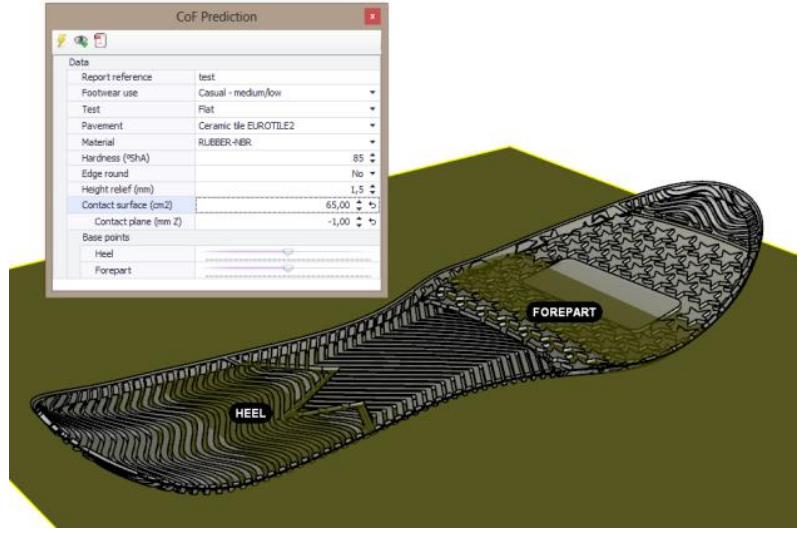

Fig. 57 - Calculation of the coefficient of friction

Once the design of the model is complete, manufacturing problems may arise. For example, it may be necessary to modify the design of the sole because the last used to manufacture the shoe has been changed. In this case, to avoid completely redesigning the sole, a sole deformation is applied in accordance with the change to the last (see Figures 59 and 60).

The hollow area created in the initial design has been designed so that it can be used as a reconfigurable element, and this latter has been created in such a way that it confers grip depending on the use of the footwear. Other possibilities include 3D printing of this element by the user for aesthetic purposes (see Figure 61).

As can be seen in Table 1, the complete model was designed using the tools presented here in a total of 1 hour and 10 minutes, representing a $60 \%$ reduction in the average time taken to design a sole similar to the one shown.

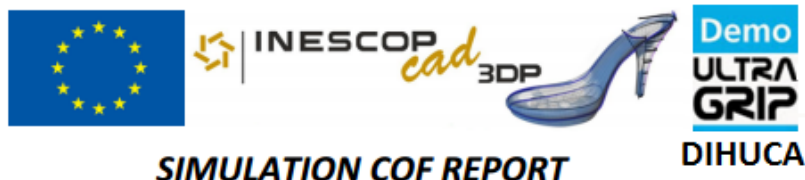

Report reference:test

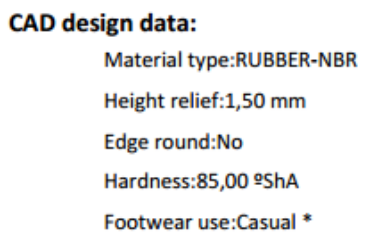

*Technical Report CEN ISO/TR 20880:2007, Footwear. Requeriments of footwear components. Soles.

Simulation data slip test (CEN-ISO 13287:2012):

Pavement type:Ceramic tile EUROTILE2

Test type:FLAT

Contact surface: $65,00 \mathrm{~cm} 2$

CoF value:0,44

Validation:VALID

Fig. 58 - Final report

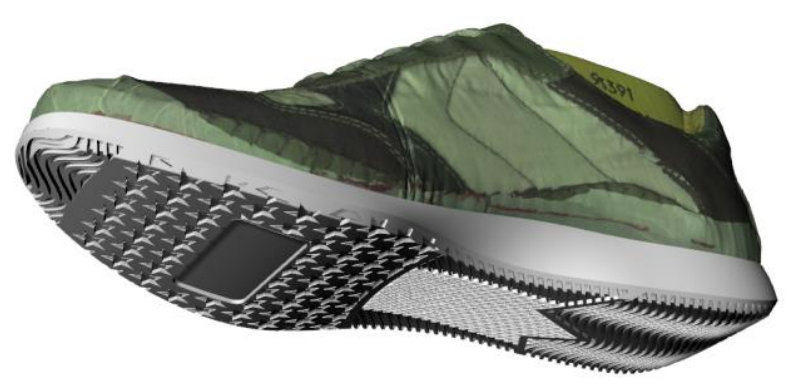

Fig. 59 - Sole prototype and last

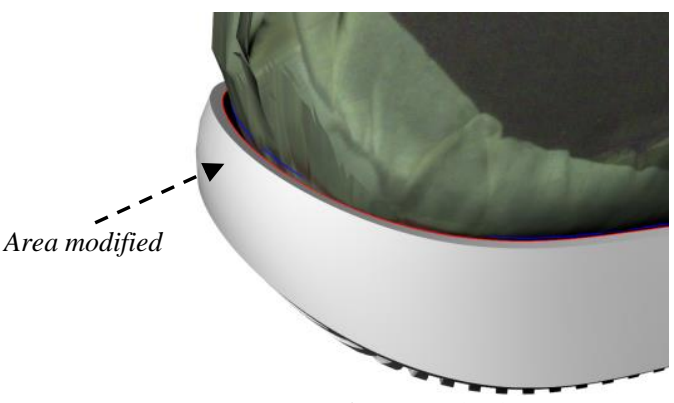

Fig. 60 - Fit in heel area 


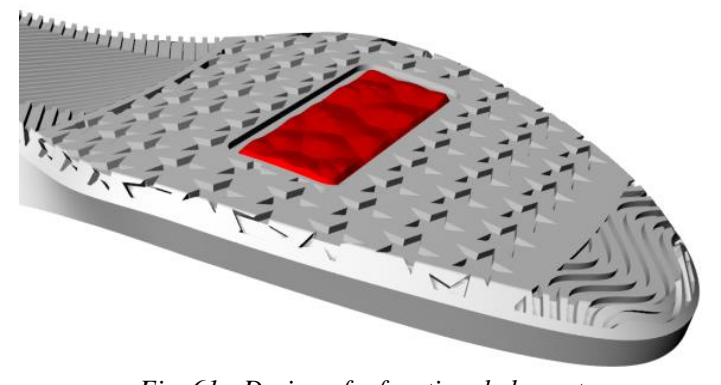

Fig. 61 - Design of a functional element

\begin{tabular}{l|c}
\hline Action & Time \\
\hline Creation of master lines. & $20 \mathrm{~min}$ \\
Creation of parametric links. & $15 \mathrm{~min}$ \\
$\begin{array}{l}\text { Design of 2D tread and transfer to the sole } \\
\text { body. }\end{array}$ & $15 \mathrm{~min}$ \\
Creation of 3D tread. & $5 \mathrm{~min}$ \\
Simulation. Slip resistance. & $5 \mathrm{~min}$ \\
Final fitting. & $5 \mathrm{~min}$ \\
Design of reconfigurable element. & $5 \mathrm{~min}$ \\
\hline
\end{tabular}

\section{Discussion and conclusions}

The footwear industry needs new CAD methodologies and tools in order to tackle the sector's emerging challenges of customisation and quality. Here, we have described a specific design methodology that facilitates this process in the shoe sole manufacture sector. Our proposal has involved the design of CAD tools that make it possible to reduce design time and increase sole quality and functionality. These tools include the parametric design of the main sole body, the outsole tread and functional elements. In addition, they encompass polysurface parameterisation to create complex 3D designs, and geometry deformation to make final adjustments to the sole models.

Furthermore, our proposal also uses mathematical models to simulate slip resistance and obtain the shoe sole's coefficient of friction, incorporating this functionality in the design workflow and thus allowing designers to make decisions in the early stages of product development.

The methodology described here conforms to the usual shoe sole design workflow, and can be used in different phases of the design and manufacturing process. Furthermore, initial implementation causes very little disruption.

Similarly, we have shown that additive manufacturing offers new possibilities for the sector, making it possible to manufacture highly complex designs. However, we have not addressed the issues inherent to the additive manufacturing process, namely the requirements that 3D geometries for printing must meet and the constraints of $3 \mathrm{D}$ printing machines. These generate problems such as perfect closure of the polygonal meshes comprising the geometries, or correct structure of meshes, which will be considered in future research.

\section{Acknowledgements}

Financial support from IVACE (Instituto Valenciano de Competitividad Empresarial) Project: DIHUCA - Complex tread designs for footwear soles (IMDEEA/2015/4).

\section{References}

[1] ElMaraghy H, AlGeddawy T, Azab A, ElMaraghy W (2012) Change in manufacturing - research and industrial challenges. Enabling Manufacturing Competitiveness and Economic Sustainability, Springer Berlin Heidelberg, pp 2-9.

[2] National Research Council US (1998) Visionary Manufacturing Challenges for 2020. National Academy Press, Washington, DC.

[3] Amann K (2010) The Growing Importance of Simulation \& Analysis in Product Development. CIMDATA, FR2010-NAFEMS, Paris, France.

[4] Monticolo D, Hilaire V, Gomes S, Koukam A (2008) A multi-agent system for building project memories to facilitate the design process. Integrated Computer-Aided Engineering, 15(1)3:20.

[5] Prassad N, Plaza E (1996) Corporate memories as distributed case libraries. Proceedings of Knowledge Acquisition Workshop, Banff, AB, Canada, pp 4041 and 40-19.

[6] Dreisbach R (2010) Leveraging simulation for competitive advantage. Proceedings of the International NAFEMS Conference, Paris, France, pp 27 41.

[7] Pratt MJ, Anderson BD, Ranger T (2005) Towards the standardized exchange of parameterized feature based CAD models. Computer-Aided Design, 37(12)1251-1265.

[8] Requicha AAG, Voelcker HB (1983) Solid modelling: current status and research directions. IEEE Computer Graphics and Applications, (3)25-37.

[9] Kalay Y (1989) Modelling Objects and Environments. Wiley, New York.

[10] Kho Y, Garland M (2005) Sketching mesh deformations. Proceedings of the 2005 symposium on Interactive 3D graphics and games (I3D ‘05). ACM, pp 147-154.

[11] Chang E, Jenkins OC (2006) Sketching, articulation and pose for facial meshes. Proceedings of the 2006 ACM SIGGRAPH/Eurographics symposium on Computer animation (SCA '06), pp 271-280.

[12] Blanco R, Oliveira M (2008) Instant mesh deformation. Proceedings of the 2008 symposium on Interactive 3D graphics and games (I3D ‘08). ACM, pp $71-78$.

[13] Davia M, Jimeno-Morenilla A, Salas F (2013) Footwear bio-modelling: an industrial approach. International Journal of Computer Aided Design, 45(12):1575-1590

[14] Ju T, Schaefer S, Warren J (2005) Mean value coordinates for closed triangular meshes. ACM Transactions on Graphics (TOG), 24(3):561-566.

[15] Floater M, Kos G, Reimers M (2005) Mean value coordinates in 3d. Computer Aided Geometric Design, 22(7):623-631.

[16] Joshi P, Meyer M, DeRose T (2007) Harmonic coordinates for character articulation. ACM Transactions on Graphics (TOG), 26(3):71

[17] Lipman Y, Kopf J, Cohen-Or D, Levin D (2007) GPU-assisted positive mean value coordinates for mesh deformations. Proceedings of the fifth Eurographics symposium on Geometry processing (SGP '07). Eurographics Association, pp 117-123.

[18] Lipman Y, Levin D, Cohen-Or D (2008) Green coordinates. ACM Transactions on Graphics (TOG), 27(3):78:1-78:10

[19] Igarashi T, Moscovich T, Hughes JF (2005) As-rigid-as-possible shape manipulation. Proceedings of Siggraph'05, ACM TOG 24, (3):1134-1141.

[20] Sorkine O, Alexa M (2007) As-rigid-as-possible surface modelling. Proceedings of Eurographics Symposium on Geometry Processing, pp 109116.

[21] AM Platform (2014). Additive Manufacturing: Strategic Research Agenda.

[22] Sheng Y, Yaoyao FZ (2015) Additive manufacturing-enabled design theory and methodology: a critical review. International Journal of Advanced Technology Manufacturing, 80(1-4):327-342.

[23] Bikas H, Stavropoulos P, Chryssolouris G (2015) Additive manufacturing methods and modelling approaches: a critical review. International Journal of Advanced Manufacturing Technology, online pp 1-17.

[24] ULTRAGRIP (FP7-SME-2010-1,262413) www.ultragrip.eu

[25] DEMOULTRAGRIP (FP7-SME-2013-3, 606549) www.demoultragrip.eu

[26] Cherrez LE, Rico, ALH (2014) Línea de calzado masculino con construcción strobel en las tallas 38 al 42 (Doctoral dissertation, in Spanish).

[27] Aristides AG, Requicha J, Rossignac R (1992) Solid Modelling and Beyond. IEEE Computer Graphics and Applications, 12(5):31-44.

[28] Huang S, Liu P, Mokasdar A, Hou L (2003) Additive manufacturing and its societal impact: a literature review. International Journal of Advanced Manufacturing Technology, 67(5):1191-1203. 